\title{
A unified approach to estimate land and water reflectances with uncertainties for coastal imaging spectroscopy
}

\author{
David R. Thompson ${ }^{\mathrm{a}, *}$, Kerry Cawse-Nicholson ${ }^{\mathrm{a}}$, Zachary Erickson ${ }^{\mathrm{b}}$, Cédric G. Fichot ${ }^{\mathrm{c}}$, \\ Christian Frankenberg ${ }^{\mathrm{b}}$, Bo-Cai Gao ${ }^{\mathrm{d}}$, Michelle M. Gierach ${ }^{\mathrm{a}}$, Robert O. Green ${ }^{\mathrm{a}}$, Daniel Jensen ${ }^{\mathrm{a}, \mathrm{e}}$, \\ Vijay Natraj $^{\mathrm{a}}$, Andrew Thompson ${ }^{\mathrm{b}}$ \\ a Jet Propulsion Laboratory, California Institute of Technology, Pasadena, CA 91109, USA \\ ${ }^{\mathrm{b}}$ California Institute of Technology, Pasadena, CA 91125, USA \\ ${ }^{\mathrm{c}}$ Boston University, Boston, MA 02215, USA \\ ${ }^{\mathrm{d}}$ Naval Research Laboratory, Washington, DC 20375, USA \\ ${ }^{\mathrm{e}}$ Department of Geography, University of California at Los Angeles, Los Angeles, CA 90095, USA
}

\section{A R T I C L E I N F O}

\section{Keywords:}

Imaging spectroscopy

Coastal and inland waters

Optimal estimation

PRISM

Atmospheric correction

Bio-optical models

Statistical methods

Portable remote imaging SpectroMeter

Hyperspectral imaging

\begin{abstract}
A B S T R A C T
Coastal ecosystem studies using remote visible/infrared spectroscopy typically invert an atmospheric model to estimate the water-leaving reflectance signal. This inversion is challenging due to the confounding effects of turbid backscatter, atmospheric aerosols, and sun glint. Simultaneous estimation of the surface and atmosphere can resolve the ambiguity enabling spectral reflectance maps with rigorous uncertainty quantification. We demonstrate a simultaneous retrieval method that adapts the Optimal Estimation (OE) formalism of Rodgers (2000) to the coastal domain. We compare two surface representations: a parametric bio-optical model based on Inherent Optical Properties (IOPs); and an expressive statistical model that estimates reflectance in every instrument channel. The latter is suited to both land and water reflectance, enabling a unified analysis of terrestrial and aquatic domains. We test these models with both vector and scalar Radiative Transfer Models (RTMs). We report field experiments by two airborne instruments: NASA's Portable Remote Imaging SpectroMeter (PRISM) in an overflight of Santa Monica, California; and NASA's Next Generation Airborne Visible Infrared Imaging Spectrometer (AVIRIS-NG) in an overflight of the Wax Lake Delta and lower Atchafalaya River, Louisiana. In both cases, in situ validation measurements match remote water-leaving reflectance estimates to high accuracy. Posterior error predictions demonstrate a closed account of uncertainty in these coastal observations.
\end{abstract}

\section{Introduction}

Remote imaging spectrometers, also known as hyperspectral imagers, can map ecosystem composition over wide areas. They are wellsuited for the land/water boundary comprised of coastal oceans, inland water, and wetlands, which provide invaluable societal services and are sensitive to climate change (Hartmann et al., 2013; ESAS, 2018; Parslow et al., 2000). Imaging spectrometers in the Visible/Shortwave Infrared (VSWIR) range can map the benthic composition (Hochberg et al., 2003), sessile, emergent and near-shore vegetation (Turpie et al., 2015), and water properties such as suspended sediment (Fichot et al., 2016) or phytoplankton (Kudela et al., 2015). Spatial sampling near $30 \mathrm{~m}$ enables detailed study of these phenomena (Giardino et al., 2018). A recent survey by the National Academies advocated an orbital spectroscopic investigation with global coverage of the coastline (ESAS,
2018). However, investigators will only observe the radiance at the instrument, so they must account for atmospheric interference.

Coastal and inland waters can hinder current atmospheric correction methods (Wang et al., 2010; Goyens et al., 2013; Palacios et al., 2015). Transitional zones have optically complex waters with indeterminacy between turbidity, atmospheric aerosol, and glint. The community has developed atmospheric correction algorithms for turbid scenarios (Mobley et al., 2016), but many are multi-band methods that do not extend naturally to spectroscopic data. Their accuracy could be improved (Goyens et al., 2013) and their relative performance is unpredictable for novel conditions. Their assumptions may fail for partlyinundated pixels, high turbidity, or high spatial resolution that invalidates Cox-Munk glint models (Muller-Karger et al., 2018). The algorithms vary in statistical rigor, use of climatology or other background knowledge, and consideration of noise or calibration

\footnotetext{
* Corresponding author.

E-mail address: david.r.thompson@jpl.nasa.gov (D.R. Thompson).
} 


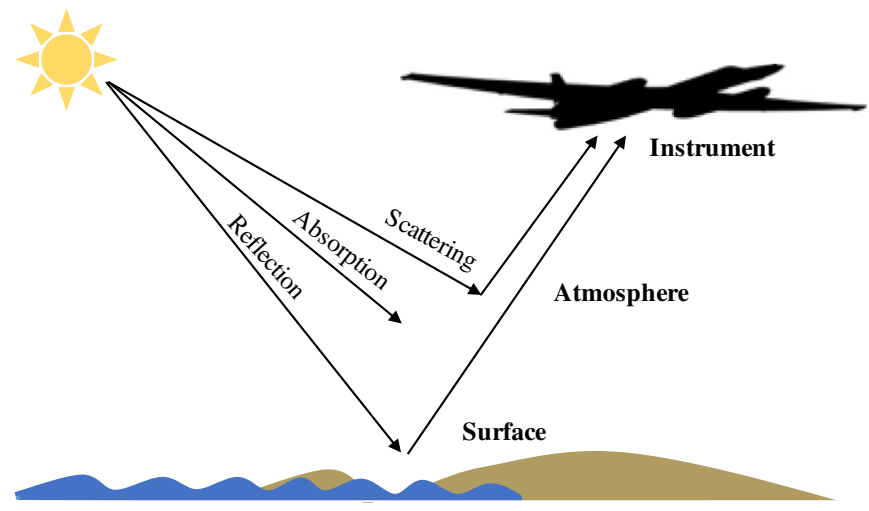

Fig. 1. The retrieval models the entire observation system consisting of surface, atmosphere, and instrument. Aircraft image courtesy NASA.

uncertainties. Uncertainty accounting will be important to prevent bias from local atmospheric conditions in global studies. Measurement uncertainty is significant due to weak reflectance signals and difficult radiometric calibration (Helmlinger et al., 2016). More broadly, the community lacks a unified approach for atmospheric correction to study interactions between land and sea.

New algorithms offer a potential solution. Recently, Thompson et al., (2018c) adapted the Optimal Estimation (OE) formalism of Rodgers, (2000) for imaging spectroscopy with a physics-based model combining surface, atmosphere, and instrument (Fig. 1). While that work used terrestrial spectra, $\mathrm{OE}$ also promises advantages for coastal applications. First, it exploits combined surface/atmosphere models using the full spectral range and resolution. This implicitly captures surface slope, shape, and magnitude constraints to disambiguate atmospheric effects, and is valuable for atmospheric aerosols lacking distinctive absorption signatures. It incorporates climatology using statistical priors. These surface and atmosphere constraints allow the retrieval to fully utilize the rich information from the diverse coastal spectrum. Rather than define such constraints explicitly using ad hoc heuristics, OE captures them automatically in the statistical properties of the combined model. $\mathrm{OE}$ is also distinct from other simultaneous open water retrieval methods (Wang et al., 2010) which apply mainly to multi-band data or limited water properties (Frouin et al., 2019), often disregard uncertainties, and do not generally work over land.

A second advantage of $\mathrm{OE}$ is flexibility to use different surface parameterizations, including but not limited to bio-optical models. Parametric bio-optical models specify the water leaving reflectance using a small number of optical parameters. For example, Lee et al., 2002 use Inherent Optical Properties (IOPs) having direct physical interpretations as - for example - Colored Dissolved Organic Matter (CDOM) and phytoplankton pigment absorption. Alternatively, more expressive parameterizations such as those of our prior work (Thompsonet al., 2018c) retrieve reflectance separately in each channel. This uses a larger state vector to estimate wholly novel reflectance shapes. It can represent unanticipated phenomena such as new benthic reflectance signatures or subtle phytoplankton pigmentation signals (Gitelson et al., 2011). More generally, it enables a unified methodology for aquatic, terrestrial. and inland water surfaces.

OE's third advantage for coastal studies is rigorous uncertainty propagation and accounting. It incorporates priors on instrument performance, meteorology, and water properties from ancillary measurements or climatology (Rodgers, 2000). It enables posterior predictive uncertainties of retrieved surface and atmosphere parameters for subsequent analyses. This allows a principled fusion of data from multiple locations and times. It also reveals information content and the state vector parameters measurable from the observing system.

This work extends imaging spectrometer Optimal Estimation to coastal and inland water domains. We describe bio-optical and channelwise surface parameterizations, quantify the remote measurement information, and evaluate consistency of uncertainties with field measurements. We also address the interplay between different surface and atmospheric parameters including aerosols. This demonstration of surface models and Bayesian estimation is independent from the specific choice of atmospheric Radiative Transfer Model (RTM). We evaluate both scalar and vector RTMs to show that the framework is general enough to incorporate any atmospheric physics assumptions.

The experiments use two airborne case studies from different coastal environments. First, we consider NASA's Portable Remote Imaging SpectroMeter, PRISM (Mouroulis et al., 2014), an imaging spectrometer that measures the visible to near infrared (VNIR) interval. PRISM flew on a high-altitude ER-2 aircraft over Santa Monica Bay, CA, in October 2015 , an observation which viewed $95 \%$ of the atmospheric column making it a direct analogue of orbital observations. Coincident in-situ validation spectra corroborate reflectance accuracy. Second, we analyze data from NASA's Airborne Visible Infrared Imaging Spectrometer, AVIRIS-NG (Thompson et al., 2018a), which covers the entire visible to shortwave (VSWIR) interval. In October 2016, AVIRIS-NG observed the Wax Lake Delta and lower Atchafalaya River, LA (Jensen et al., 2018), a network of turbid rivers with high backscatter and suspended sediment loads. In both cases, we demonstrate in situ measurements are consistent with remote OE retrievals.

Section 2 reviews atmospheric correction methods, focusing on coastal spectroscopy. Section 3 details $\mathrm{OE}$ and our implementation for the coastal ocean. Sections 4 and 5 present two field experiments. Each description summarizes the instrument preparation, acquisition, and the ancillary data collection. We compare surface parameterizations and performance versus traditional methods.

\section{Background}

Atmospheric correction of VSWIR remote sensing is a broad field. The algorithms partition naturally into categories for multi-band or spectroscopic instruments, and terrestrial or oceanic environments. Multi-band methods include the MODIS approach for land (Vermote et al., 2002) and ocean (Wang and Shi, 2007). They typically follow a sequential strategy of: first, estimating atmospheric parameters using simplified surface assumptions; and then, after the atmosphere is fixed, algebraically inverting the measured radiance to estimate the channelwise surface reflectance. They estimate atmospheric features with specific band values, hard-coded ratios, and thresholds based on physical insight and hard-coded climatological constraints (Schläpfer et al., 1998). A typical example is the aerosol retrieval algorithm of Kaufman et al., 1997 which exploits reduced particle scattering in near infrared to predict the reflectances of dark pixels. Discrepancies, attributed to aerosol scattering, permit retrievals based on a global climatology partitioned by latitude and longitude. The designer optimizes thresholds and boundaries using instrument characteristics and considerable background knowledge. After estimating the atmosphere, the waterleaving reflectance can be recovered algebraically with a functional relationship for gaseous and particulate transmission and absorption. Examples include formulae by Vermote et al. (1997) and Vermote and Kotchenova (2008) for land, or Gordon and Clark (1981) and Gordon and Wang (1994) for ocean This inversion uses optical coefficients from Radiative Transfer Models (RTMs) (Kotchenova et al., 2008) such as the MODTRAN package (Berk et al., 2014) based on the scalar DISORT code (Stamnes et al., 1988), or the VLIDORT-based 6S package (Kotchenova et al., 2006; Kotchenova and Vermote, 2007), based on the vector VLIDORT code (Spurr, 2006).

The open ocean deserves special attention, since water-leaving radiance is a small fraction of the total signal reaching the sensor. Moreover, key productivity indicators like chlorophyll absorption are sensitive to distortion by atmospheric scattering. On the other hand, the water optical system enables some simplifications. For example, strong liquid water absorption means path radiance dominates in the near 
infrared (Siegel et al., 2000), or, for very turbid waters, the shortwave infrared (Wang and Shi, 2007). Another advantage of the deep ocean is relative insensitivity to atmospheric gases such as $\mathrm{H}_{2} \mathrm{O}$. Finally, at spatial resolutions relevant to the open ocean, many unwanted surface effects such as Fresnel reflectance and sunglint are predictable by windspeed-based models (Gordon, 2005).

Unfortunately, coastal environments thwart many of these advantages. Their water surfaces have higher variability and turbidity (Parslow et al., 2000; Neumann et al., 2000). They do not always satisfy the dark near infrared assumption (Wang and Shi, 2007). Higher spectral resolution is needed for signatures such as functional type indicators, benthic environments or near shore vegetation. Moreover, their higher spatial resolutions may impact Cox-Munk glint models. For the interested reader, Wang et al., 2010 review ocean color atmospheric correction; Hochberg et al., (2015) review coastal applications.

Fewer atmospheric correction algorithms have been developed for spectroscopic observations with hundreds of channels. VSWIR spectra are sensitive to atmospheric effects ranging from extreme Rayleigh and Aerosol scattering in the ultraviolet, through pervasive water vapor effects throughout the near infrared and into deep water absorptions at 1480 and $1880 \mathrm{~nm}$. Reviews are found in Thompson et al., 2018c for land and Frouin et al. (2019) for open ocean. Most approaches are variations of multi-band sequential algorithms (Guanter et al., 2009). Common software packages include ATREM (Gao et al., 1993), ATCOR (Richter and Schläpfer, 2005; Richter and Schläpfer, 2002), and FLAASH (Perkins et al., 2012). Specific coastal versions have been developed to use dark-NIR aerosol retrievals (Gao et al., 2000; Gao et al., 2007; Thompson et al., 2017). Variants have been deployed to atmospherically-correct data from the HICO instrument (Lewis et al., 2009; Corson et al., 2008). Nevertheless, it is still common to rely on empirical, in-scene corrections such as the empirical line approach (Thompson et al., 2016) or cloud-shadow methods (Amin et al., 2014). Such variants require manual intervention and homogeneous atmospheric effects throughout the scene, making them infeasible for global applications.

A separate class of retrieval methods deserves special attention. Simultaneous algorithms estimate both atmospheric and surface parameters simultaneously in a combined solution, applying constraints to the surface and/or atmosphere to resolve numerical indeterminacy. This can potentially make retrievals more robust to departures from heuristic surface assumptions (Frouin et al., 2019). The joint surface/ atmosphere fit facilitates rigorous posterior uncertainty predictions (Frouin and Pelletier, 2015). Most simultaneous algorithms apply to multi-band instruments. Examples include the POLDER/PARASOL algorithm, which adjusts water surface and atmosphere parameters to optimize a spectral match between model and measurement by a multiband, multi-angle observer (Dubovik et al., 2011). In the open ocean, Steinmetz et al. (2011) demonstrate a Principal Component (PC) surface representation enabling joint retrieval of sunglint, aerosol, and water properties. Brajard et al. (2008) and Brajard et al. (2012) adopt a similar method for multi-band observations of water using a neural network as the radiative transfer model. Saulquin et al. (2016) use a Gaussian mixture model to represent surfaces in a small number of bands. Finally, Frouin and Pelletier (2015) have demonstrated Bayesian retrievals with informed priors to permit simultaneous retrieval and posterior uncertainty propagation for open water.

While these surface/atmosphere parameterizations are effective for multi-band instruments, future coastal spectroscopy will demand both a high degree of flexibility in the surface (to capture subtle perturbations related to different phytoplankton pigments and benthic cover) as well as high dimensionality (to accommodate hundreds of spectral channels). It is still unclear whether prior methods can satisfy these objectives (Frouin et al., 2019). On land, surfaces are even more diverse and target signatures even more subtle. There, joint surface/atmosphere fitting has been implemented only for restricted surface models (Hou et al., 2016; Hou et al., 2017). The full potential of simultaneous fitting
Table 1

Notation conventions. Boldface indicates vectors, vector-valued functions, and matrices. We treat all vectors as columns for matrix algebra.

\begin{tabular}{|c|c|}
\hline Symbol & Interpretation \\
\hline A & Averaging kernel matrix, per Rodgers (2000) \\
\hline a & Optical absorption coefficient of the water column, $\mathrm{m}^{-1}$ \\
\hline $\mathbf{b}_{b}$ & Optical backscatter coefficient of the water column \\
\hline $\mathbf{c}_{i}$ & Vector-valued correction factors for pushbroom column $i$ \\
\hline$d(j)$ & Distance of a surface reflectance to the cluster $j$ \\
\hline$e_{o}$ & Solar irradiance at the top of the atmosphere \\
\hline f & Forward model, a vector-valued function mapping $\mathbf{x}$ to $\mathbf{y}$ \\
\hline$g_{0}, g_{1}$ & coefficients relating backscatter and absorption to $r_{r s}$ \\
\hline G & Gain matrix, with elements $\partial x / \partial y$ \\
\hline$G$ & $\begin{array}{l}\text { Colored Dissolved Organic Matter (CDOM) absorption, as in Lee et al. } \\
(2004)\end{array}$ \\
\hline I & Identity matrix \\
\hline $\mathbf{K}$ & Jacobian matrix, with elements $\partial y / \partial x$ \\
\hline$\ell_{o b s}$ & Measured radiance at the sensor \\
\hline$\ell_{o b s}{ }^{*}$ & "True" radiance at the sensor aperture \\
\hline$m$ & Number of measurement channels in $\ell_{\text {obs }}$ \\
\hline$n$ & Number of elements in the state vector $\mathbf{x}$ \\
\hline$P$ & Phytoplankton absorption, as in $P$ of Lee et al. (2004) \\
\hline$q$ & Quality score quantifying pushbroom striping \\
\hline$Q$ & Sunglint magnitude in units of water-leaving reflectance \\
\hline $\mathbf{r}_{r s}$ & $\begin{array}{l}\text { Remote sensing reflectance below the air-water interface (per } \\
\text { steradian) }\end{array}$ \\
\hline $\mathbf{R}_{r s}$ & $\begin{array}{l}\text { Remote sensing reflectance above the air-water interface (per } \\
\text { steradian) }\end{array}$ \\
\hline s & Spherical sky albedo at the surface \\
\hline$\widehat{\mathbf{s}}$ & Posterior covariance of state vector \\
\hline $\mathbf{S}_{a}$ & Covariance of state vector prior distribution \\
\hline $\mathbf{S}_{b}$ & Covariance of uncertainty due to model unknowns \\
\hline$S_{h}$ & Covariance of surface state (prior distribution) \\
\hline $\mathbf{S}_{j}$ & Covariance of surface state (prior distribution) for cluster $j$ \\
\hline $\mathbf{S}_{L I B}$ & Covariance of reflectance library spectra \\
\hline $\mathbf{S}_{m}$ & Covariance of resolving error component of $\widehat{\mathbf{S}}$ \\
\hline $\mathbf{S}_{n}$ & Covariance of measurement error component of $\widehat{\mathbf{S}}$ \\
\hline $\mathbf{S}_{s}$ & Covariance of atmospheric state (prior distribution) \\
\hline $\mathbf{S}_{y}$ & Covariance of measurement noise due to the instrument \\
\hline $\mathbf{S}_{y i}$ & Covariance of measurement noise at cross-track location $i$ \\
\hline$S_{\epsilon}$ & Covariance of random observation noise \\
\hline $\mathbf{t}$ & Diffuse and direct transmission of the sun/surface/sensor path \\
\hline $\mathbf{x}$ & Complete state vector, $x=\left[\mathbf{x}_{S U R F}, \mathbf{x}_{A T M}\right]$ \\
\hline$\widehat{\mathbf{x}}$ & Estimated state vector \\
\hline $\mathbf{x}_{a}$ & Mean of state vector prior distribution \\
\hline $\mathbf{x}_{s}$ & Mean of surface state vector prior distribution \\
\hline $\mathbf{x}_{h}$ & Mean of atmospheric state vector prior distribution \\
\hline $\mathbf{x}_{j}$ & Mean of surface state vector prior distribution for cluster $j j$ \\
\hline $\mathbf{x}_{A T M}$ & Free parameters of atmosphere \\
\hline $\mathbf{x}_{\text {SURF }}$ & Free parameters of surface \\
\hline $\mathbf{x}_{V I S}$ & Visibility at $550 \mathrm{~nm}(\mathrm{~km})$ \\
\hline$\widehat{\mathbf{x}}$ & Estimated state vector \\
\hline$X$ & Backscatter, as in $X$ of Lee et al., 2004 \\
\hline $\mathbf{y}$ & Radiance measurement in each channel, $\mu \mathrm{W} \mathrm{nm} \mathrm{m}^{-1} \mathrm{~cm}^{-2} \mathrm{sr}^{-1}$ \\
\hline$\hat{\mathbf{y}}$ & Modeled radiance in each channel, $\mu \mathrm{W} \mathrm{nm}{ }^{-1} \mathrm{~cm}^{-2} \mathrm{sr}^{-1}$ \\
\hline$Y$ & Backscatter exponent, as in $Y$ of Lee et al., 2004 \\
\hline$z$ & L1 norm (area under the curve) of a reflectance spectrum \\
\hline$\alpha$ & Regularizer for shrinkage covariance estimation \\
\hline e & Random observation noise \\
\hline$\chi^{2}$ & Cost function \\
\hline$\rho_{o b s}$ & Top of Atmosphere (TOA) reflectance \\
\hline$\rho_{a}$ & Atmospheric path reflectance \\
\hline$\rho_{s}$ & Surface water-leaving reflectance \\
\hline$\hat{\rho}_{s}$ & Estimated surface reflectance \\
\hline$\phi_{o}$ & Cosine of solar zenith angle \\
\hline$\sigma_{\ell}$ & Noise-equivalent change in radiance \\
\hline$\sigma_{C A L}$ & Standard deviation of radiometric uncertainty \\
\hline$\hat{\sigma}$ & Standard deviation of marginal posterior uncertainty \\
\hline$\sigma_{n}$ & Standard deviation of marginal posterior measurement noise \\
\hline$\sigma_{m}$ & Standard deviation of marginal posterior resolving error \\
\hline$\sigma_{\lambda}$ & Vector of systematic radiative transfer uncertainty \\
\hline$\circ$ & Element-wise multiplication \\
\hline / & Element-wise division (for matrices and vectors) \\
\hline
\end{tabular}


and the potential benefits for uncertainty quantification has yet to be realized operationally. The Optimal Estimation (OE) formalism of Rodgers (2000) offers one promising solution across both aquatic and land targets. The following section describes the approach and presents an implementation adapted for the coastal aquatic domain.

\section{Optimal estimation approach}

\subsection{Mathematical background}

We build on a decades-long heritage of $\mathrm{OE}$ in atmospheric instruments like OCO-2 (Cressie, 2018). OE explains the measured radiance $y$ by modeling the observation system including the surface, atmosphere and instrument. The free parameters to be estimated from a state vector $\mathbf{x}=\left[x_{1}, \ldots, x_{n}\right]^{T}$. Table 1 summarizes our notation, following conventions in Rodgers (2000) and Thompson et al. (2018a), (2018c). Boldface symbols indicate vectors, vector-valued functions, and matrices. Vectors are lowercase and matrices are uppercase. The hat symbol $\wedge$ denotes an estimated quantity. We treat all vectors as columns for matrix algebra. Our state vector is the concatenation of free surface parameters $\mathbf{x}_{\text {SURF }}$ and free atmospheric parameters $\mathbf{x}_{A T M}$, detailed in Sections 3.2 and 3.3 respectively. A physics-based forward model $\mathbf{f}(\mathbf{x})$ predicts the instrument radiance measurement for any state using any Radiative Transfer Model (RTM) compatible with the instrument wavelength range and sampling. The actual measurement $\boldsymbol{y}$ incurs zero-centered random noise $\mathbf{\epsilon}$ :

$\mathbf{y}=\mathbf{f}(\mathbf{x})+\epsilon$ for $\mathbf{x}=\left[\mathbf{x}_{S U R F}, \mathbf{x}_{A T M}\right]^{T}$

The algorithm begins with an initial guess for $\mathbf{x}$. This state has a probability proportional to the product of a statistical prior representing background knowledge, and a likelihood term representing the goodness of fit between the model and the measurement. We adjust the state vector repeatedly in the gradient direction of increasing posterior probability, iterating until convergence. At each step, we linearize the forward model about the current solution. Locally, we treat both prior and likelihood terms as multivariate Gaussian distributions. The $\log$ probability is a cost $\chi^{2}$ with two squared error terms penalizing the model's divergence from the measurement and the prior:

$\chi^{2}(\mathbf{x})=\frac{1}{2}\left(\mathbf{x}-\mathbf{x}_{a}\right)^{T} \mathbf{S}_{a}^{-1}\left(\mathbf{x}-\mathbf{x}_{a}\right)+\frac{1}{2}(\mathbf{y}-\mathbf{f}(\mathbf{x}))^{T} \mathbf{S}_{\epsilon}^{-1}(\mathbf{y}-\mathbf{f}(\mathbf{x}))$

The prior distribution has mean $\mathbf{x}_{a}$ and covariance matrix $\mathbf{S}_{a}$. The covariance matrix $\mathbf{S}_{\boldsymbol{\epsilon}}$ represents observation uncertainty; it incorporates a measurement noise covariance $\boldsymbol{S}_{\boldsymbol{y}}$ and unknown, unretrieved parameters of the surface or atmosphere that are treated as random variables with a covariance $\boldsymbol{S}_{b}$. Typically $\mathbf{S}_{b}$ has diagonal structure; we translate it to measurement covariance using the Jacobian matrix $\mathbf{K}_{b}$, the partial derivatives of the radiance with respect to each unknown:

$\mathbf{S}_{\epsilon}=\mathbf{S}_{y}+\mathbf{K}_{b} \mathbf{S}_{b} \mathbf{K}_{b}^{T}$

Similarly, the Jacobian matrix $\mathbf{K}$ gives partial derivatives of $\mathbf{f}(\mathbf{x})$ with respect to $\boldsymbol{x}$, i.e. $K_{i j}=\partial y_{i} / \partial x_{j}$. It reveals the forward model's sensitivity to the state vector and is used in the gradient descent calculation. Leastsquares optimization methods such as the Levenberg-Marquardt algorithm (More, 1978) converge quickly to a cost minimum. Multiple restarts could prevent sub-optimal local minima, but were not needed for our experiments where the error surface was stable near the solution state. The prior distribution provides additional resilience to local minima. (Thompson et al., 2018c)provide additional detail.

In summary, Optimal Estimation for imaging spectroscopy requires:

1. A surface parameterization $\mathbf{x}_{\text {SURF }}$ representing retrieved surface parameters.

2. A surface prior, i.e. the mean and covariance structure of surface parameters. These form elements in $\mathbf{x}_{a}$, and a diagonal block in $\mathbf{S}_{a}$.

3. An atmosphere parameterization $x_{A T M}$ representing retrieved atmospheric parameters.

4. An atmospheric prior, i.e. the mean and covariance atmospheric parameters. These define the other elements in $\mathbf{x}_{a}$ and $\mathbf{S}_{a}$.

5. A forward model $\mathbf{f}(\mathbf{x})$, a deterministic operation mapping the free parameters to the at-sensor radiance with a Radiative Transfer Model (RTM).

6. The measurement covariance $S_{y}$, representing the random instrument noise.

7. The covariance of model unknowns $S_{b}$, representing variability in unretrieved model parameters.

8. Jacobian matrices $\boldsymbol{K}$ and $\boldsymbol{K}_{\boldsymbol{b}}$ formed respectively by partial derivatives of radiance with respect to the retrieved and unretrieved model parameters, reevaluated at the current solution with each iteration.

This structure provides considerable design flexibility. In particular, designers can include any quantity in the state vector regardless of whether the measurement has information to estimate it reliably. Rodgers, 2000 gives several ways to characterize the actual information content. The gain matrix $\mathbf{G}$ is the sensitivity of the retrieved state to the measurement $\boldsymbol{y}$, defined as $G_{i j}=\partial \widehat{x}_{i} / \partial y_{j}$. It has a closed form expression:

$\mathbf{G}=\left(\mathbf{K}^{T} \mathbf{S}_{\epsilon}^{-1} \mathbf{K}+\mathbf{S}_{a}^{-1}\right)^{-1} \mathbf{K}^{T} \mathbf{S}_{\epsilon}^{-1}$

The averaging kernel matrix $\mathbf{A}=\mathbf{G K}$ is the sensitivity of the retrieved state vector to the "true" state vector, accounting for instrument noise and correlations between surface and atmosphere. Its diagonal elements show the Degrees of Freedom (DOF) for each state vector parameter (Rodgers, 2000). A DOF near unity means that the retrieved state tracks the true value. A DOF near zero indicates low sensitivity. Rows of the $A$ matrix are averaging kernels that show the result's sensitivity to different elements of the true state. They portray the resolving power in the retrieval process

Under local linearity, the posterior predictive uncertainty has covariance $\widehat{\mathbf{S}}$ :

$\widehat{\boldsymbol{S}}=\left(\mathbf{K}^{T} \mathbf{S}_{\epsilon}^{-1} \mathbf{K}+\mathbf{S}_{a}^{-1}\right)^{-1}$

This posterior distribution accounts for several uncertainties. One is observation error from random instrument noise or unknown parameters of the environment that are not retrieved. This has a covariance $\mathbf{S}_{n}$. Another is the intrinsic information in the inversion process relative to the prior, caused by measurement insensitivity to the parameters of interest. This source has a covariance matrix $\mathbf{S}_{m}$. The two combine via:

$$
\begin{gathered}
\widehat{S}=G C_{\in} G^{T}+(I-A) S_{a}(I-A)^{T} \\
=S_{n}+S_{m}
\end{gathered}
$$

This decomposition can quantify the sources of retrieval uncertainty. More generally, the posterior error predicts system performance enabling comparison of different physics assumptions and observing conditions.

\subsection{Surface parameterizations and prior distributions}

The term surface reflectance can refer to several distinct physical properties (Schaepman et al., 2009). This work deals with the hemispherical directed reflectance representing the reflected fraction of incident light from the entire hemisphere above the surface, measured in the direction of the sensor. This is the starting point for most analyses of the Earth surface and underlies "Level 2" science data products in prior campaigns. We evaluate two alternative parameterizations of $\boldsymbol{x}_{S U R F}$. The first uses the water column's Inherent Optical Properties (IOPs). The second extends the statistical surface model of Thompson et al., (2018c) to the aquatic domain, providing a common formalism for atmospheric correction across land and aquatic zones that can handle highly turbid inland water. 


\subsubsection{Bio-optical representation}

Our first parameterization uses the bio-optical model of Lee et al. (1999) and Lee et al. (2002). It relates the angular remote-sensing reflectance below the air/water interface, $\boldsymbol{r}_{r s}$, to the remote sensing reflectance above the water surface, $\boldsymbol{R}_{r s}$, and the water leaving reflectance $\rho_{s}$ :

$\rho_{s} / \pi \sim \mathbf{R}_{r s}$, where $\quad \mathbf{r}_{r s}=\frac{\mathbf{R}_{r s}-Q}{1.562\left(\mathbf{R}_{r s}-Q\right)+0.518}$ for $Q \geq 0$

Here, $Q$ is a spectrally-constant value representing the magnitude of additive sun glint. There are many functional forms relating $\mathbf{r}_{r s}$ to column properties like optical absorption, $\mathbf{a}$, and backscatter, $\mathbf{b}_{b}$ (Lee et al., 2002; Lee et al., 2004; Gordon et al., 1988). We favor the following simple relation for optically deep water:

$r_{r s}=g_{1} \frac{\mathbf{b}_{b}}{\mathbf{a}+\mathbf{b}_{b}}+g_{0}\left(\frac{\mathbf{b}_{b}}{\mathbf{a}+\mathbf{b}_{b}}\right)^{2}$

with $g_{0}=0.0895$ and $g_{1}=0.1247$ (Lee et al., 2002). The spectrum $\mathbf{b}_{b}$ is the sum of water backscatter $\mathbf{b}_{w}$ and particle backscatter $\boldsymbol{i}_{b p}$; we define the former as constant (Morel, 1974) and the latter as in Lee et al. (1999):

$\mathbf{b}_{b p}=X\left(\frac{400.0}{\lambda}\right)^{Y}$ for $\quad X \geq 0, \quad 0 \leq Y \leq 2.5$

We retrieve the $Y$ and $X$ as free parameters. Here, $\boldsymbol{\lambda}$ represents the center wavelength of each channel's spectral response in nanometers.

The absorption spectrum $\mathbf{a}$ is the sum of absorption coefficients by water, $\mathbf{a}_{w}$, phytoplankton, $\mathbf{a}_{\Phi}$, and CDOM + detritus $\mathbf{a}_{g}$, using the following formulae,

$\mathbf{a}=\mathbf{a}_{w}+\mathbf{a}_{\Phi}+\mathbf{a}_{g}$

$\mathbf{a}_{g}=G e^{-0.015(\lambda-440)}$ for $G \geq 0$

$\mathbf{a}_{\Phi}=\Phi_{1} P+\Phi_{2} P \log P$ for $P \geq 0$

where $G$ and $P$ are free parameters related to CDOM + detritus absorption and phytoplankton respectively. Coefficients $\Phi_{1}$ and $\Phi_{2}$ referenced in Lee et al. (1999) describe phytoplankton absorption signatures. A good approximation for the physical quantity of chlorophylla in units of $\mathrm{mg} \mathrm{m}^{-3}$ is Lee et al. (1999):

$\mathrm{Chl}-\mathrm{a}=e^{(\log (P / 0.06) / 0.65)}$

Finally, we model $F$, a feature variously attributed to backscatter and the emission of phytoplankton fluorescence. This free parameter is a nonnegative "fluorescence line height" in units of water-leaving radiance. It defines a Gaussian shape with center wavelength $683 \mathrm{~nm}$ and standard deviation of $10.6 \mathrm{~nm}$, following Abbott and Letelier (1999).

In summary, the IOP parameterization generates a water-leaving reflectance spectrum using six free parameters: glint $Q$, the backscatter $X$, the backscatter exponent $Y$, the CDOM + detritus absorption $G$, the phytoplankton absorption $P$, and the fluorescence line height $F$. For simplicity we take these parameters to be unconstrained and uncorrelated, ascribing a prior covariance matrix with large diagonal values.

\subsubsection{Channelwise representation}

We also consider a more expressive parameterization based on the statistical approach of Thompson et al., (2018c). It represents separate reflectance values for each instrument channel. A multivariate Gaussian prior constrains the values to physically-plausible solutions. The designer can use statistical regularization to create strong or weak constraints on reflectance shape. Typically, we use very weak constraints to ensure that the measurement dominates the prior for estimating arbitrary spectral reflectance shapes. This preserves unexpected features or subtle band position shifts that are critical for many Earth science analyses. Constraints on relative slopes or discontinuities are only needed in areas with critical atmospheric information such as the oxygen A band or certain near infrared water vapor absorption features. Specific investigations can apply more constraints; for example, "tighter" Gaussians give more numerical leverage to estimate subtle atmospheric perturbations.

We define a multi-component, multivariate Gaussian prior by fitting a diverse library of reflectance spectra. This approach has precedent for surface/atmosphere inversions of MERIS multiband water measurements (Saulquin et al., 2016), making it a promising option for high resolution spectroscopy. At run time, for each retrieval iteration, we find the component Gaussian that is closest to the current state according to Mahalanobis or Euclidean distance (Thompson et al., 2018c). The closest component becomes the prior surface distribution for that iteration. The prior is normalized so it only constrains the shape but not the magnitude of the reflectance spectra. During the retrieval, we normalize each estimated reflectance spectrum before calculating distances, and after finding the best matching component, rescale it for consistency with the actual state vector.

Unlike many land surfaces, the apparent reflectance of coastal waters can have strong directionality. Our model captures the most important physical mechanisms that contribute to this non-Lambertian behavior. An additional state vector element represents the magnitude of additive sun glint, as in the $Q$ parameter of the IOP version. Its prior is unconstrained and uncorrelated from the other parameters. Second, the channelwise surface prior represents the apparent water-leaving reflectance as measured in the viewing direction, implicitly accounting for the directionality of the upwelling signal from the water column. An investigator inverting $\rho_{s}$ to retrieve water column optical parameters could use a directional model, or limit the applicable range of geometries and water conditions as in Eq. (8). A third effect, directional surface-atmosphere coupling via multiple scattering events (Fan et al., 2017), is less significant for our experiments due to low-AOT conditions and near-nadir viewing angles. Section 6 describes how the retrieval could use a combined surface/atmosphere model in the future.

Our model includes both terrestrial and aquatic components, attempting to span the space of physically-realizable surface spectra. We fit the terrestrial components with a diverse library of surfaces described in prior work (Thompson et al., 2018c). The water-leaving reflectances come from two sources. First, we construct synthetic spectra with varied backscatter, phytoplankton, and dissolved carbon content using the bio-optical model of Section 3.2.1. These cover water surfaces with low turbidity. We also incorporate a library of highly turbid inland water cases by Tan et al. (2016), extending their wavelength ranges into the shortwave infrared by extrapolating from AVIRIS-NG river water observations in our field experiment. We identify the best-matching AVIRIS-NG reflectance observation for each library ()spectrum using the spectral angle distance, and rescale the former to match at the $900 \mathrm{~nm}$ join point. This procedure proves adequate for a generic and highly regularized prior, fully encompassing the land and water surfaces in our datasets.

After assembling the library, we identify component centers using $\mathrm{K}$-means, estimate their full covariances, and finally regularize with a "shrinkage" addition to diagonal elements (Theiler, 2012). Figs. 2, 3, and 4 illustrate the mean spectra and marginal standard deviations of a multi-component surface model. The distribution of sunglint-removed spectra captures patterns of NIR and SWIR water reflectance slopes and magnitudes. We find results are stable with respect to the number of components and regularization.

\subsubsection{Atmospheric parameterization, prior distributions, and RTM simulations}

The atmospheric model uses a physics-based simulation of photon transport in the atmosphere. To demonstrate generality of the OE approach we consider two different Radiative Transfer Model (RTM) options. We focus mainly on the MODTRAN 6.0 RTM which is widely used by the coastal and terrestrial imaging spectroscopy communities, and 


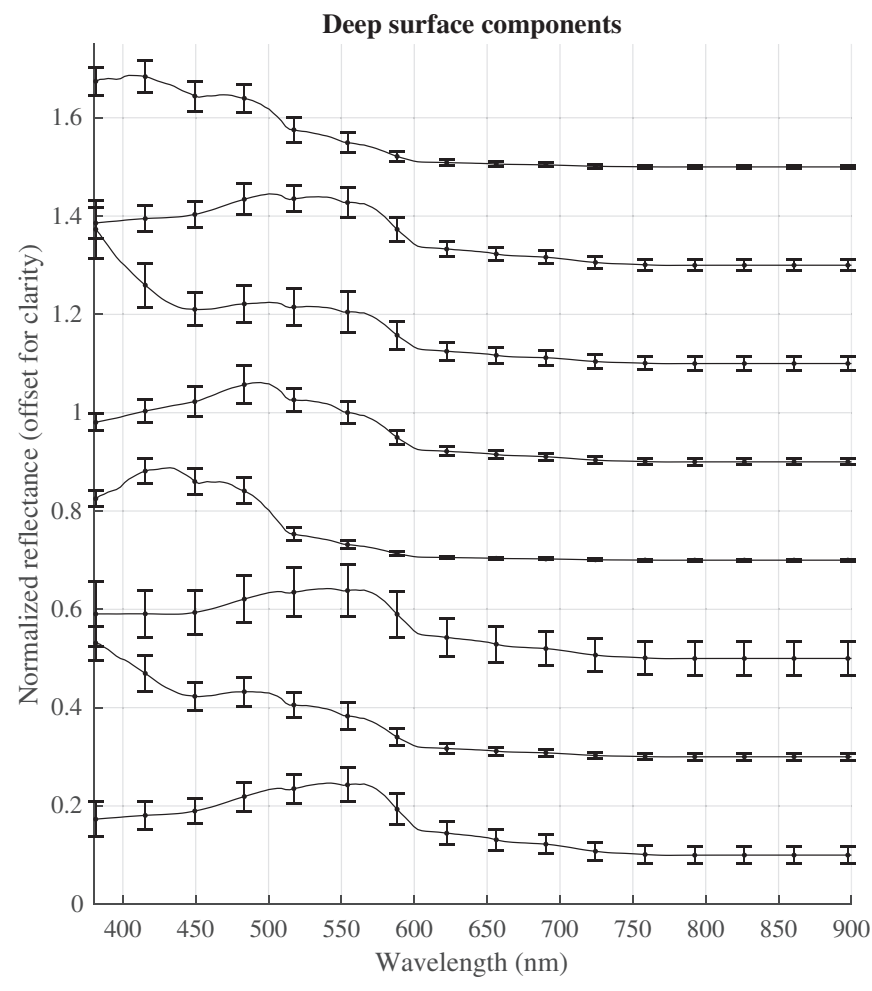

Fig. 2. Deep aquatic components of the statistical surface model, showing means and marginal standard deviations of the covariance matrix. Large standard deviations near $1000 \mathrm{~nm}$ relate to calibration challenges in these wavelengths.

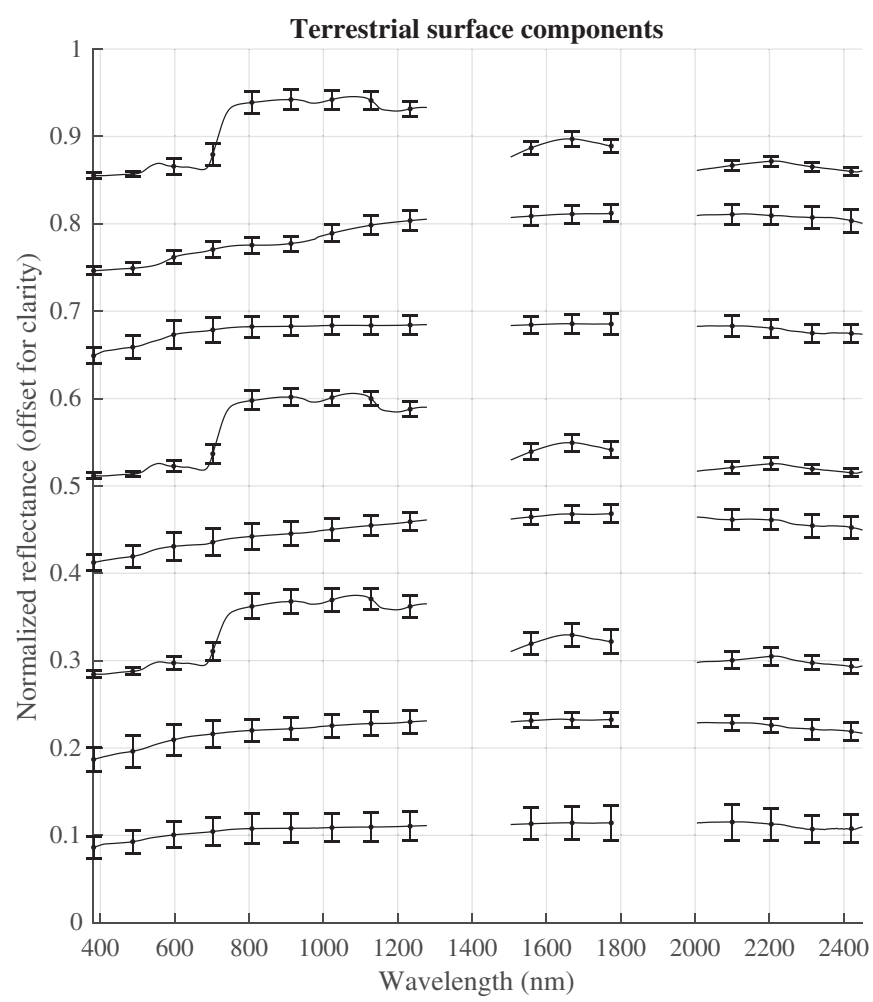

Fig. 3. Terrestrial components of the statistical surface model, showing means and marginal standard deviations of the covariance matrix.

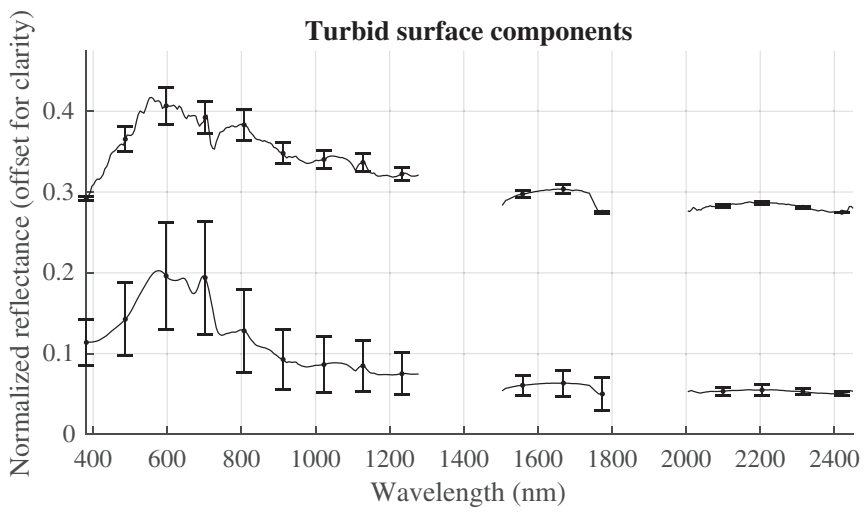

Fig. 4. Turbid components of the statistical surface model, showing means and marginal standard deviations of the covariance matrix.

incorporated into typical commercial packages such as FLAASH (Perkins et al., 2012) and ATCOR (Richter and Schläpfer, 2005; Richter and Schläpfer, 2002). However, coastal observations of dark targets are sensitive to minor differences in Rayleigh and aerosol scattering calculations which may require vector-based RTMs that respect polarization. To demonstrate that these models are also an option for the $\mathrm{OE}$ approach, we perform a limited subset of tests using the 6SV radiative transfer engine (Kotchenova et al., 2006; Kotchenova and Vermote, 2007).

Most atmospheric parameters are known a priori (such as observation geometry) or sufficiently constrained by climatology (such as the atmospheric profile). Consequently, our atmospheric state vector has just four free parameters. We retrieve the total water vapor column abundance, in $\mathrm{g} \mathrm{cm}^{-2}$. We also retrieve the Aerosol Optical Depth (AOD) at $550 \mathrm{~nm}$. The MODTRAN RTMs permit arbitrary scattering and absorption profiles, so we retrieve the AOD independently for each of three canonical aerosol types: an absorbing carbon aerosol; a dust aerosol characteristic of rural or continental sources; and a highly scattering sulfate aerosol type. We do not intend these retrieved AODs to imply the mixture of species observed, but instead use them as a diverse palette for the retrieval to represent aerosol-like distortions. In other words, they act as a structured error term promoting accurate surface retrievals without restriction to a stock aerosol mixture. In the 6SV RTM simulations, we use a stock marine model. Retrieved AODs were quite low for all field experiments making the specific choice of aerosol optical properties less significant.

We write the measured radiance $\mathbf{y}$ as $\ell_{o b s}=\left[\ell_{\lambda 1}, \ldots, \ell_{\lambda m}\right]^{T}$, and transform it to an apparent top of atmosphere reflectance $\rho_{o b s}$. This normalizes solar input to remove variability downward irradiance $e_{\boldsymbol{o}}$ at the top of the atmosphere (Kneizys et al., 1988). It includes $\phi_{o}$ representing the cosine of the solar zenith angle. OE permits optical coupling between surface and atmosphere, but we use a decoupled approximation for simplicity and heritage from existing VSWIR codes like ATCOR, FLAASH, TAFKAA and ATREM. The spectrum $\rho_{o b s}$ decomposes into a path reflectance $\rho_{\boldsymbol{a}}$, a spherical albedo $\mathbf{s}$, atmospheric transmittance $\mathbf{t}$, and the surface reflectance $\rho_{s}$ (Adler-Golden et al., 1999; Thompson et al., 2018c):

$\rho_{o b s}=\frac{\ell_{o b s} \pi}{\phi_{o} \mathrm{e}_{\mathrm{o}}}=\rho_{\boldsymbol{a}}+\frac{\text { to } \rho_{\boldsymbol{s}}}{1-\left(\mathbf{s} \circ \rho_{s}\right)}$

Here, $\circ$ indicates element-wise multiplication. Since $\rho_{s}$ represents the water leaving reflectance as measured in the sensor direction, our surface models must capture the full expected range of bi-directional reflectances. The channelwise parameterization handles this implicitly in the probability density function, which captures the range of upwelling radiance from the water. In contrast, the IOP parameterization promotes invariance by limiting applicability to a range of conditions (Lee et al., 2002). Directional IOP models would extend applicability at 
the cost of additional complexity. Both parameterizations handle the sunglint contribution to apparent reflectance through the explicit sunglint variable. Diffuse skylight is implicit in the spherical albedo term of the atmospheric model. Working in the space of apparent reflectances, there is no direct representation of downwelling and upwelling radiances, but those could be calculated by independent RTM runs based retrieved atmospheric properties.

Our model accounts for the pushbroom instrument in which different Focal Plane Array (FPA) elements see different optical paths and scattering geometries. We use the RTMs to calculate $\mathrm{e}_{\boldsymbol{o}}, \mathbf{t}, \rho_{\boldsymbol{a}}$, and $\mathbf{s}$ for a set of viewing geometries and reference atmospheres, with lookup table interpolation to find intermediate values. We then downsample by the instrument response function. Denoting resampling by brackets $<>$ and free coefficients as functions of the state vector:

$\widehat{\boldsymbol{y}}=\left\langle\phi_{o} \mathrm{e}_{\boldsymbol{o}} \pi^{-1} \circ\left[\rho_{\boldsymbol{a}}(\mathbf{x})+\frac{\mathbf{t}(\mathbf{x}) \circ \rho_{\boldsymbol{s}}(\mathbf{x})}{\left.1-\mathbf{s}(\mathbf{x}) \circ \rho_{\boldsymbol{s}}(\mathbf{x})\right)}\right]\right\rangle$

The only unspecified atmospheric terms are water vapor and AOD. Atmospheric parameters are unconstrained and uncorrelated with the other state variables.

\section{Field demonstration I: PRISM coastal observations of Santa Monica Bay, CA}

\subsection{Experimental method}

We evaluate two different environments: the low-sediment, phytoplankton-dominated coastal waters of Santa Monica Bay; and the highly turbid inland waters of the Wax Lake Delta and lower Atchafalaya River. Together, the study areas have diverse water properties with two different remote instruments and a number of in situ comparisons comparable to, or larger than, prior coastal spectroscopy studies (Palacios et al., 2015).

We first evaluate the retrieval using a PRISM flight on a high altitude ER-2 aircraft over Santa Monica Bay, California, on October 262,015. Santa Monica is a near-shore bay in the Southern California Bight that is semi-enclosed and influenced by the populated Los Angeles basin (Trinh et al., 2017). It is an interesting test case due to its high productivity and diverse aquatic ecosystem (Hickey, 1992; Corcoran and Shipe, 2011). Nutrients come from various sources including seasonal upwelling as well as transient point sources such as wastewater outflow during diversion events (Trinh et al., 2017). The ER-2 overflight took place during one such wastewater diversion. Remote and in situ measurements reveal a large algal bloom associated with this outflow event (Trinh et al., 2017). The ER-2 flight altitude of $20 \mathrm{~km}$ provides wide area coverage of this feature at $20 \mathrm{~m}$ ground sampling. It observes approximately $95 \%$ of the atmospheric column making it an analogue for orbital measurements. Fig. 5 shows the flightline with the location of coincident in situ validation spectra that were acquired using
Satlantic HyperPro instruments with a protocol described by Trinh et al., (2017). We reference these sites as D8W, D8.5W, D9W, and D9.5W.

PRISM measures radiance at wavelengths spanning the $370-1050 \mathrm{~nm}$ range and sampled at approximately $3 \mathrm{~nm}$. We initially calibrate the PRISM data using a laboratory measurement of a National Institute of Standards Technology (NIST) reference source imaged under known viewing geometry. We then generate an initial flat field estimate using an optical integrating sphere. We transform the raw instrument data to units of $\mu \mathrm{W} \mathrm{nm}{ }^{-1} \mathrm{sr}^{-1} \mathrm{~cm}^{-2}$, and adjust the data to account for any remaining drift in the radiometric response of different FPA elements following the procedure detailed in the appendix. We orthorectify the data cube using standard practice, ray tracing the view direction of each instrument element to a digital elevation model. We derive the instrument position from synchronized onboard IMU/GPS data. Our validation uses the average radiance of a square region approximately $100 \mathrm{~m}$ on a side centered on each in situ collection site.

We use a vicarious calibration to address post-installation differences in radiometric response. This accounts for factors including: window transmission effects for the ER-2 installation, which are expected to be significant relative to the measured signal; any mean calibration drift over time (as opposed to the relative flat field variability); implicitly, any time-dependent differences in the solar irradiance spectrum or the fine-scale spectral sampling thereof (Thompson et al., 2015a); and any deviation from a completely linear gain response that could affect results at the sub- $1 \%$ level for the dark ocean targets. This is standard practice for imaging spectrometers such as AVIRIS-NG and PRISM, which experience more thermal change than typical orbital instruments (Green et al., 1998). Here, we use the first in situ spectrum at the D8W location as the calibration target. It is still necessary to retrieve atmospheric parameters since they are not measured at the surface, and in any case, terms like the water vapor absorption along the sun-surface-observer path are not directly measurable. Consequently, we use an initial retrieval to calculate atmospheric parameters, apply them to the surface reflectance to predict the uncorrected atsensor radiance via Eq. (14), and calculate channelwise gain coefficients (scaling factors) that align the PRISM measurement. This leaves three in situ spectra separated by several kilometers as validation. In the following section, the AVIRIS-NG experiment uses a similar strategy over multiple flightlines and days to demonstrate generalization across more widely differing atmospheres, surfaces, and geometries.

Table 2 shows the uncertainty budget for both in situ and remote reflectances. The only uncertainty term for the in-situ reflectances is the temporal variability, which we calculate from the full sequence of measurements spanning the range of the overflight. We exclude calibration and measurement uncertainty, since the former is not known and the latter is not significant due to the large number of integrations.

We validate retrieved reflectances with several quality scores. First, we consider a qualitative assessment of "bowing" or parabolic magnitude trends in the cross-track direction, which reveals geometric
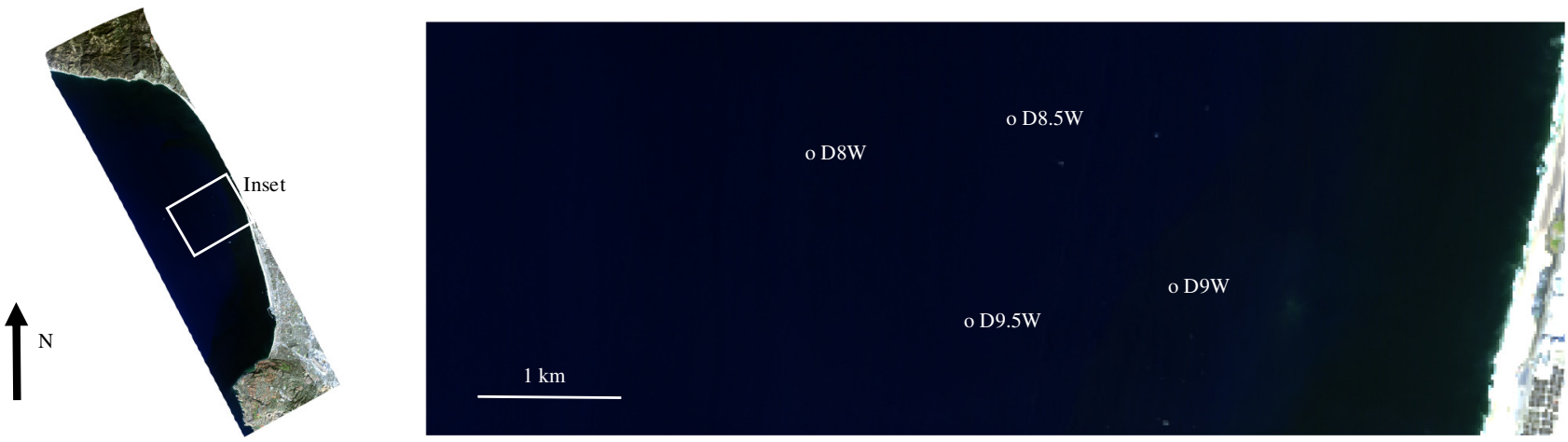

Fig. 5. Visible color image of PRISM flightline prm20151026t173213. The inset shows the location of the study area and validation sites. 
Table 2

Uncertainty account for the PRISM flightline. Uncertainty values are standard deviations, except where indicated. $\mathrm{H}_{2} \mathrm{O}$ uncertainties are consistent with Rothman et al., 2013.

\begin{tabular}{|c|c|c|c|}
\hline Source & Elements & Value & Notes \\
\hline \multicolumn{4}{|l|}{ Remote estimated state $\widehat{\mathbf{x}}$} \\
\hline \multicolumn{4}{|l|}{ Prior uncertainty $\mathbf{S}_{a}$} \\
\hline Surface reflectance & $242 \times 242$ & Empirical & Figs. 2-3 \\
\hline Atmospheric $\mathrm{H}_{2} \mathrm{O}$ & 1 & $>10 \mathrm{~g} \mathrm{~cm}^{-2}$ & \\
\hline Aerosol AOT550 & 3 & 0.05 & \\
\hline \multicolumn{4}{|l|}{ Observation noise $\mathbf{S}_{\boldsymbol{\epsilon}}$} \\
\hline $\begin{array}{l}\text { Measurement noise } \boldsymbol{S}_{y} \\
\text { Unknown variables } \mathbf{S}_{b}\end{array}$ & $242 \times 242$ & Empirical & A \\
\hline $\mathrm{H}_{2} \mathrm{O}$ absorption & 1 & $1 \%$ & See caption \\
\hline \multicolumn{4}{|l|}{ In situ reflectance } \\
\hline Temporal variability & 242 & Varies & Empirical \\
\hline
\end{tabular}

nonuniformities in path radiance. We then compare spectral shape and magnitude with in situ data using squared error and spectral angle metrics. Next, we compare surface reflectance from the bio-optical and channelwise parameterizations. We contrast their spectral fidelity and the information content of the state vector according to the Rodgers (2000) DOF analysis. To compare the models' ability to fit the radiance, we extract a large square region approximately 500 pixels on a side from the center of the flightline, and decompose the residual radiance errors into Empirical Orthogonal basis Functions (EOFs) using singular value decomposition of residual covariances. This reveals any systematic errors incurred from each surface modeling approach.

\subsection{Experimental results}

Fig. 6 shows the retrieved Rrs. for the entire PRISM scene in representative channels. This result used the statistical surface model with the MODTRAN RTM. Black lines show isobath contours at every $200 \mathrm{~m}$. Clouds in the southern portion appear blank. The images are consistent with data acquired on the same day by the Landsat spacecraft, as reported by Trinh et al. (2017). Cross-track radiance profiles suggest the influence of variable viewing geometry is significant for this scene. Fig. 7 shows the relationship between path radiance and the resulting reflectance. It shows a single horizontal profile across the focal plane array in the $460 \mathrm{~nm}$ channel, excluding the extrema to avoid influence from the FPA edge (left side) and surface phenomena (right side). The bowed shape results from view-dependent differences in scattering which are significant for the dark water surface. Scalar RTM calculations with lookup table interpolation provide sufficient accuracy to estimate the view-dependent path radiance, significantly improving the reflectance results. The bottom panel shows two actual reflectance retrievals from the flightline. The red curve uses a traditional nadir view assumption, while the black curve uses the complete geometryaware model. The geometry-aware version corrects the path radiance contribution. The post-correction uniformity suggests polarization effects are minimal, so that a scalar but geometry-aware calculation is sufficient for this flightline.

Table 3 shows the spectrum fit and reflectance performance metrics. We report the Mean Squared Error (MSE) of the radiance fits and the Spectral Angles (SAs) of the remote and in situ surface reflectances. The field measurement is more uncertain in the UV range, so we calculate spectral angles over the interval from $380 \mathrm{~nm}$ to $660 \mathrm{~nm}$. The long wavelength cutoff avoids the $683 \mathrm{~nm}$ peak in D9W, which is treated separately from the water-leaving reflectance by the bio-optical model. Smaller values are better. All surface reflectances match the relative shapes of in situ spectra to within a narrow spectral angle. The final columns show the fraction of OE MODTRAN RTM points for which discrepancies lie outside the $50 \%$ and $95 \%$ posterior predicted confidence intervals, based on the OE uncertainty model and Table 2. It also shows the associated $p$ value for the chi squared hypothesis test that the residuals are drawn from this distribution; in two of three cases, the $\mathrm{p}$ value is far greater than typical rejection thresholds (e.g. 0.05 ), indicating the uncertainty budget accounts for observed discrepancies.

Fig. 8 shows the maximum a posteriori radiance and reflectance solutions, with radiances residuals $v s$. the instrument measurement. The black line shows the retrieval from the statistical model with the MODTRAN RTM; error bars indicate posterior marginal standard deviations accounting for all instrument and retrieval uncertainty. The red line shows the statistical model with the 6SV RTM. The blue line shows the MODTRAN RTM using the bio-optical surface parameterization. The grey line shows the in situ result with standard deviations. The absolute reflectance magnitudes differ most in the case of D9.5W, though in practice it is common to observe magnitude shifts in remotely sensed spectral reflectance over coastal water targets with minimal impact on spectroscopic water property estimation algorithms based on spectral shape (Kudela et al., 2015).

Fig. 9 decomposes the radiance fitting error into orthogonal bases, or Empirical Orthogonal error Functions (EOFs). The left column shows radiance errors incurred by the bio-optical surface model. The right column shows radiance errors from the statistical (channelwise) surface model. The bio-optical model leaves far more structure in important water-leaving reflectance channels, suggesting that its limited flexibility cannot completely model the reflectance spectrum. This is not surprising; the six-parameter model is an approximation and actual spectra inevitably diverge due to modeling inaccuracies and novel constituents. The bio-optical model is a compromise that strives for physical interpretability rather than reflectance fidelity. In contrast, the statistical surface model shows only a few broad biases with shapes suggesting minor differences in glint and/or magnitude of the waterleaving reflectance signal. Systematic errors near $400 \mathrm{~nm}$, where calibration and solar input is most uncertain, dominate the residuals.

Finally, Fig. 10 explores sensitivity and uncertainty for both surface models. We first decompose the total uncertainty of our statistical surface model into noise and resolution terms as in Thompson et al. (2018a), (2018c). The top row shows the estimated reflectance for two held-out validation sites. D8.5W is located in blue water with little chlorophyll. D9W is located closer to shore and contains higher levels chlorophyll absorption and fluorescence. Both converged solutions have over 236 Degrees of Freedom (DOF), close to number of independent surface parameters (242). This indicates high sensitivity to reflectance variability in all channels. The atmospheric parameters all have DOFs exceeding 0.99. The middle row of Fig. 10 decomposes total uncertainty into measurement noise $\left(\mathbf{S}_{n}\right)$ and resolution $\left(\mathbf{S}_{m}\right)$, represented as the marginal standard deviations in each reflectance channel. D8.5W is darker at longer wavelengths, and the sensor noise component competes with resolving error over most of the retrieved spectrum. In general, measurement noise dominates over retrieval resolution as the main source of uncertainty. This reflects the heavy regularization intended to minimize bias on retrieved spectral shapes.

The final row of Fig. 14 shows cross sections of the gain matrix $\mathbf{G}$, i.e. the partial derivative of the state vector element with respect to measured radiance. We show $\mathrm{H}_{2} \mathrm{O}$, glint, and the atmospheric sulfate particle AOD550. The spectroscopic measurement provides leverage to disambiguate state vector elements which affect the radiance in similar ways, e.g. glint and aerosol path radiance. Their different effects in short wavelengths, and in atmospheric absorption features such as water vapor and the $760 \mathrm{~nm}$ oxygen A band, can disentangle surface and atmosphere. Fig. 11 shows the gain factors for surface terms of the IOP parameterization, demonstrating that the different parameterization affects the relative influence of the different radiance channels at the solution state. Similar patterns are apparent, however, with short wavelengths and the modulation of atmospheric absorption features providing the most information. The IOP surface parameters all have DOF 1.00, indicating that the measurement is sensitive to all independent parameters. The one exception is the $Y$ parameter 

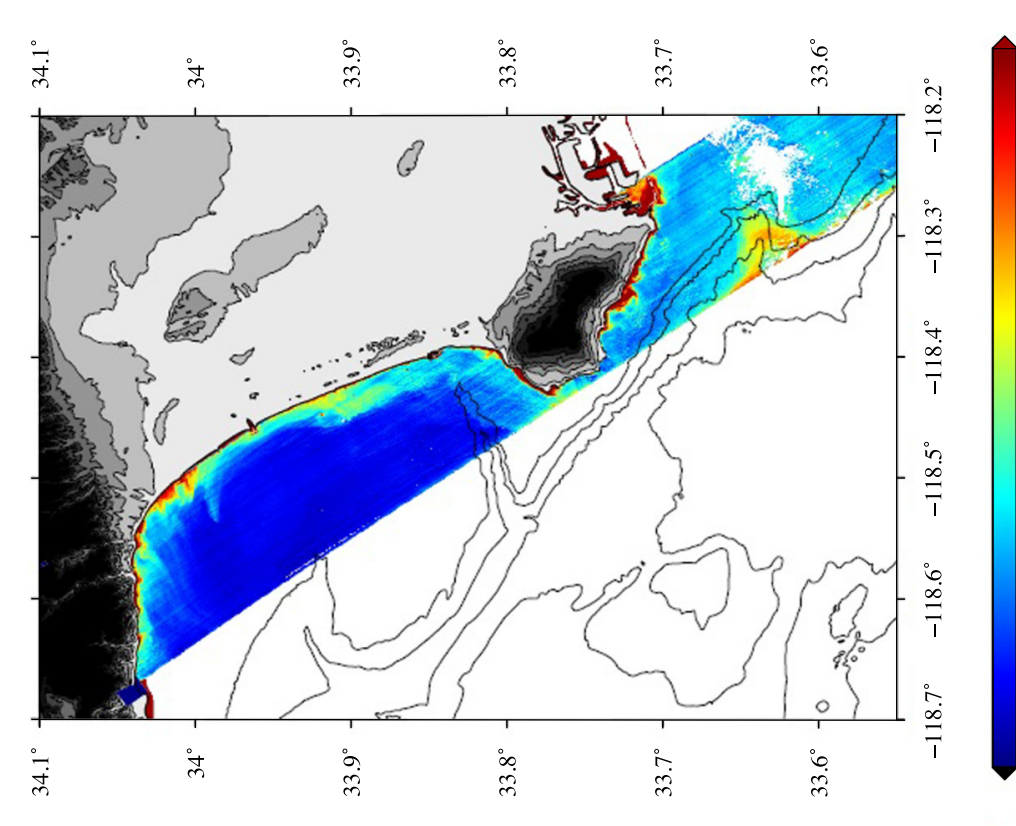

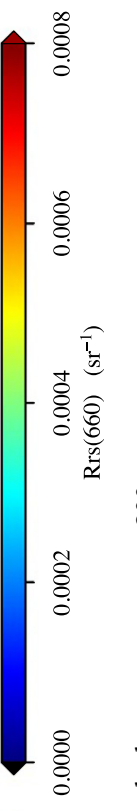

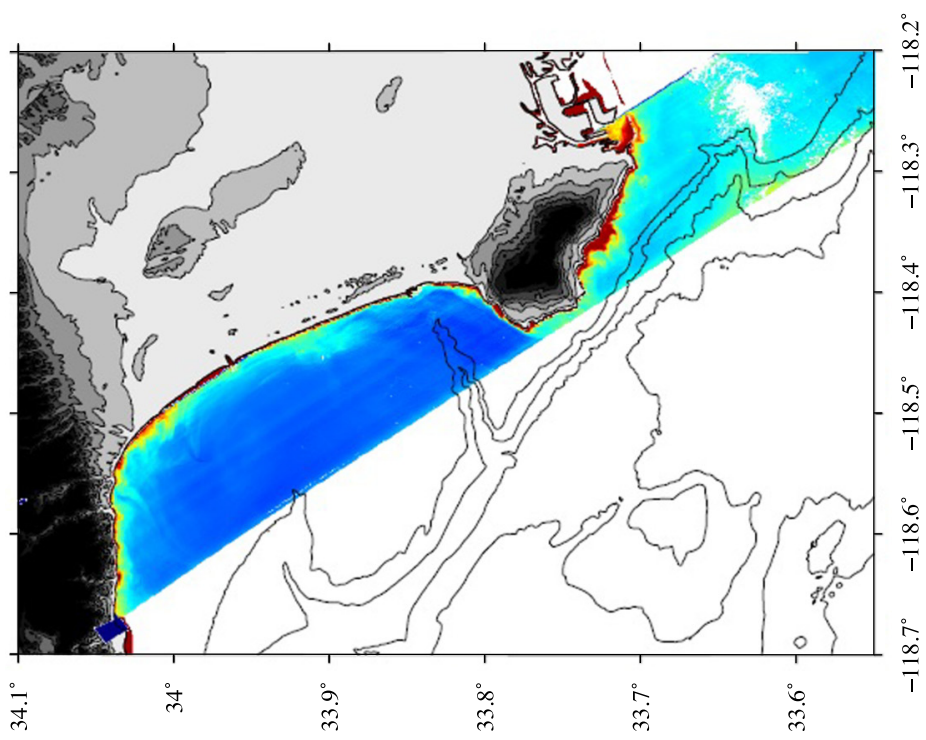

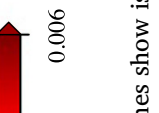

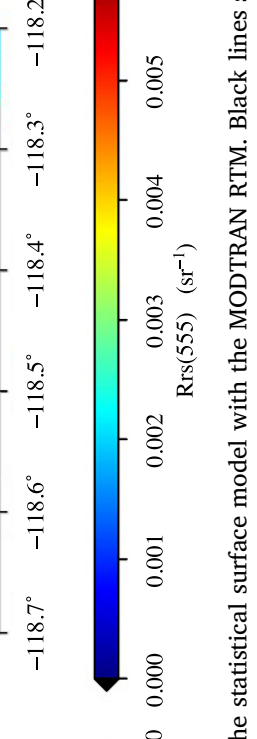

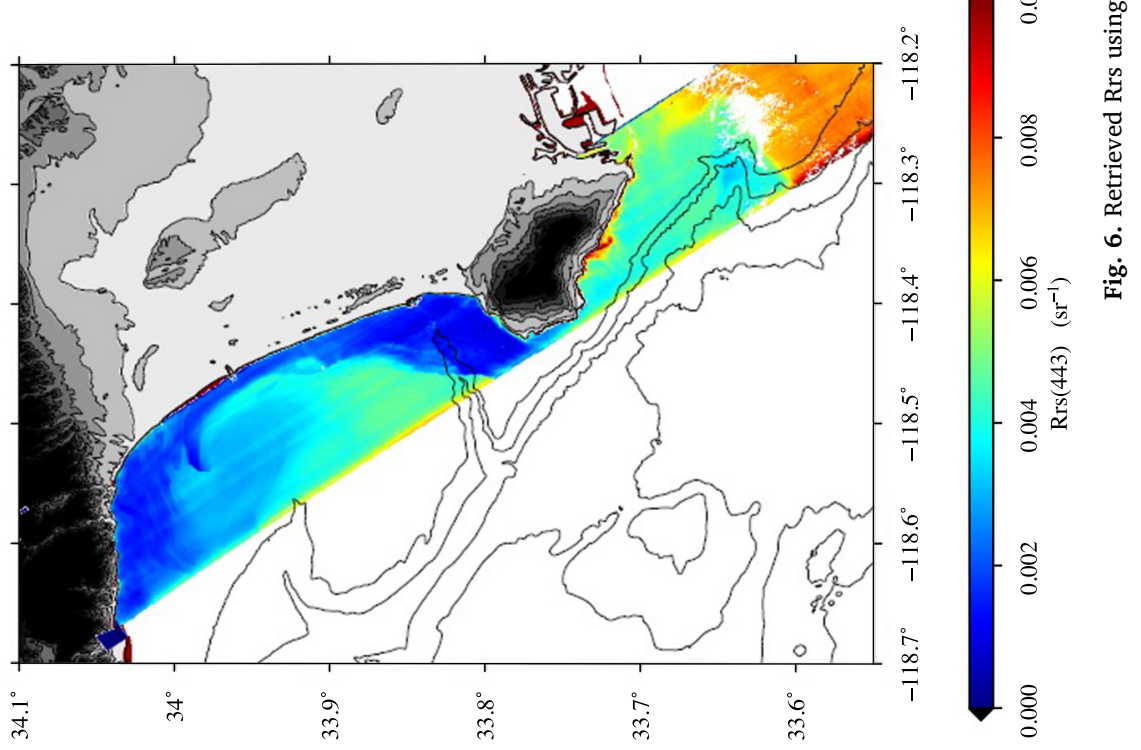



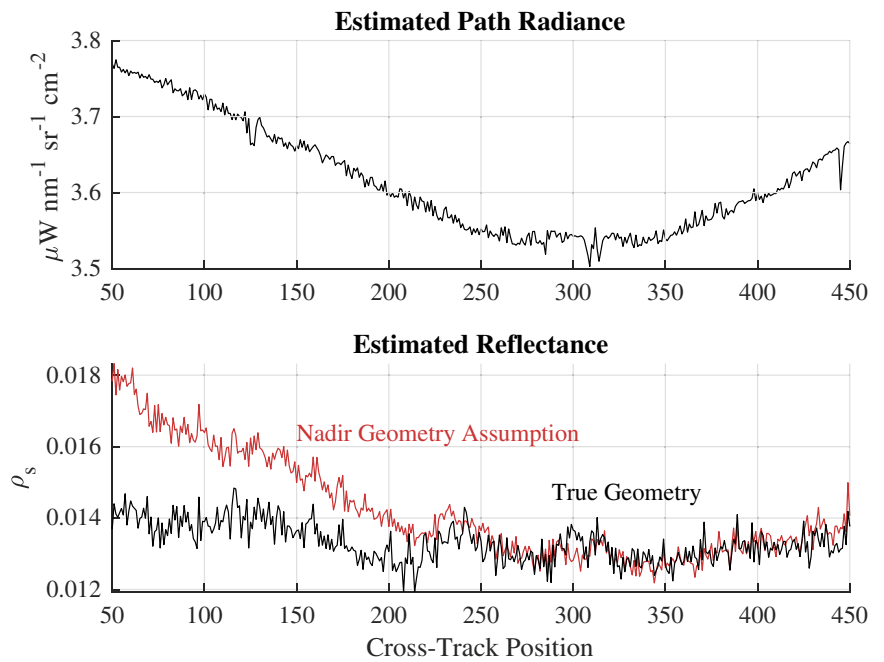

Fig. 7. The change in path radiance due to viewing geometry is significant, and proper accounting leads to a noticeable improvement in the uniformity of the reflectance. Upper and lower panels show the estimated path radiance and surface reflectance, respectively, at $460 \mathrm{~nm}$. (For interpretation of the references to color in this figure, the reader is referred to the web version of this article.)

representing the backscatter exponent, which has only $0.77 \mathrm{DOF}$. This parameter is subtle and often held fixed by inversion algorithms. Clear water cases such as these provide little leverage on particulate optical properties.

\section{Field demonstration II: AVIRIS-NG observations of the wax Lake Delta and lower Atchafalaya River, LA}

\subsection{Experimental method}

Our second field experiment is an overflight of the Wax Lake Delta Region and Atchafalaya River in Louisiana, USA by NASA's AVIRIS-NG instrument (Jensen et al., 2019). Such river delta regions are critical targets for future orbital investigations; they are diverse and productive ecosystems but vulnerable to sea level rise and climate change (Morris et al., 2002; Twilley et al., 2016). The Wax Lake Delta, the site of extensive engineering and aggradation amidst coastal sea level rise, accompanies the adjacent Atchafalaya River as the primary outflow of the Mississippi River (Twilley et al., 2016). Imaging spectroscopy can inform models of delta evolution, erosion and subsidence with measurements of river water sediment loads and the functional properties of near-shore vegetation (Jensen et al., 2019). While regional studies have made significant progress with multi-band remote sensing, empirical models based on a limited number of channels seldom generalize across regions or campaigns (Warrick et al., 2004; Chen et al., 2015). This underscores the value of full-spectrum physical models for global applicability across the land/water interface in optically complex waters.
Fig. 12 shows both mosaics of the delta area, revealing a network of turbid inland rivers and ponds.

AVIRIS-NG measures the full visible to shortwave infrared regime from 380 to $2500 \mu \mathrm{m}$, similar to the range anticipated for future investigations lik the Surface Biology and Geology observations advocated for future NASA missions (ESAS, 2018). On October 17 and 18, 2016 , a series of fligtlines transected the delta region in a dense coverage pattern over two days (Fig. 12). In this installation, a B-200 King Air aircraft platform flew at $5000 \mathrm{~m}$ altitude providing a $5 \mathrm{~m}$ ground sampling distance. Meanwhile, field teams visited different open-water locations within the river network and measured water-leaving reflectance with field radiometers. These measurements took place within approximately $6 \mathrm{~h}$ of the overflight. Field spectrometers were directed at the water surface with a nadir-pointing orientation. The team acquired multiple measurements at each site alternating with white reference calibration panels and the water surface. We exclude a handful of sites with extreme values suggesting measurement error, as well as a site in the gulf beyond the river mouth with suspect temporal stability between in situ and remote measurements. This left 11 in situ measurements. The spectral ranges of the field data varied, since both Visible/Near Infrared (VNIR) and Visible/Shortwave Infrared (VSWIR) devices were deployed. Consequently, the remote retrievals use on the full AVIRIS-NG spectral range but our in situ comparisons focus on the $380-900 \mathrm{~nm}$ range covered by all instruments.

The AVIRIS-NG analysis is similar to PRISM, starting with an initial radiometric calibration and orthorectification. To account for specular sunglint with effectively constant reflectance, we align in-situ and remote retrievals at $900 \mathrm{~nm}$ for comparison. As for PRISM, we use the first measurement as a target for vicarious calibration resulting in minor $(\sim 1 \%)$ changes to radiometric calibration coefficients. We withhold this measurement from all validations and reported results. The IOP surface parameterization does not apply to the highly turbid water so we use only the statistical option. We compare the $\mathrm{OE}$ estimation strategy to the standard AVIRIS-NG atmospheric correction result distributed by the instrument team (Thompson et al., 2015b). The standard algorithm is a conventional sequential atmospheric correction. It uses absorption coefficients derived from HITRAN 2012 (Rothman et al., 2013) together with scattering calculations by the 6S RTM (Vermote et al., 1997) to form a lookup table of optical coefficients parameterized by water vapor and pressure altitude. It retrieves these two parameters from the depths and shapes of $\mathrm{H}_{2} \mathrm{O}$ and $\mathrm{O}_{2}$ absorption features respectively. The standard product incorporates multiple empirical correction steps, including an adjustment to account for calibration uncertainty in the blue/ultraviolet interval (Thompson et al., 2018a) and a multiplicative residual suppression vector to fix systematic reflectance residuals related to RTM modeling and spectral sampling of atmospheric structure. We hold corrections close to unity and apply the same values uniformly to all spectra in the campaign. Consequently, evaluating many spectra over the course of multiple days contrasts the generalization performance of the simultaneous and conventional methods, which are $\mathrm{OE}$ and the standard approach, respectively Table 4 shows the complete uncertainty account. The AVIRIS-NG measurement uncertainty is channel- and signal-dependent,

Table 3

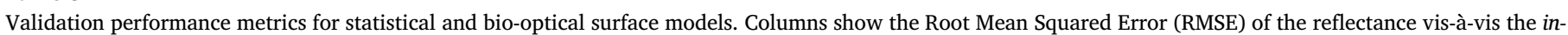

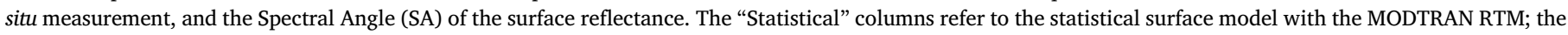

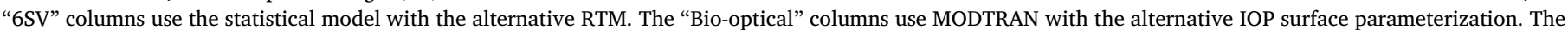

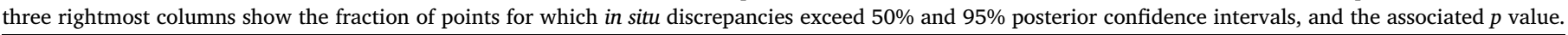

\begin{tabular}{|c|c|c|c|c|c|c|c|c|c|}
\hline \multirow[t]{2}{*}{ Trial } & \multicolumn{2}{|c|}{ Statistical (MODTRAN) } & \multicolumn{2}{|c|}{ Statistical (6SV) } & \multicolumn{2}{|c|}{ Bio-optical } & $\mathrm{CI}$ & CI & \multirow[t]{2}{*}{$p$} \\
\hline & RMSE & SA & RMSE & SA & RMSE & SA & $>50 \%$ & $>95 \%$ & \\
\hline D8.5W & 0.00050 & 0.033 & 0.00069 & 0.022 & 0.00037 & 0.034 & 10.2 & 0.0 & 1.00 \\
\hline D9W & 0.00323 & 0.100 & 0.00089 & 0.081 & 0.00254 & 0.084 & 98.3 & 56.2 & 0.00 \\
\hline D9.5W & 0.00063 & 0.041 & 0.00019 & 0.027 & 0.00048 & 0.026 & 42.6 & 0.0 & 1.00 \\
\hline
\end{tabular}



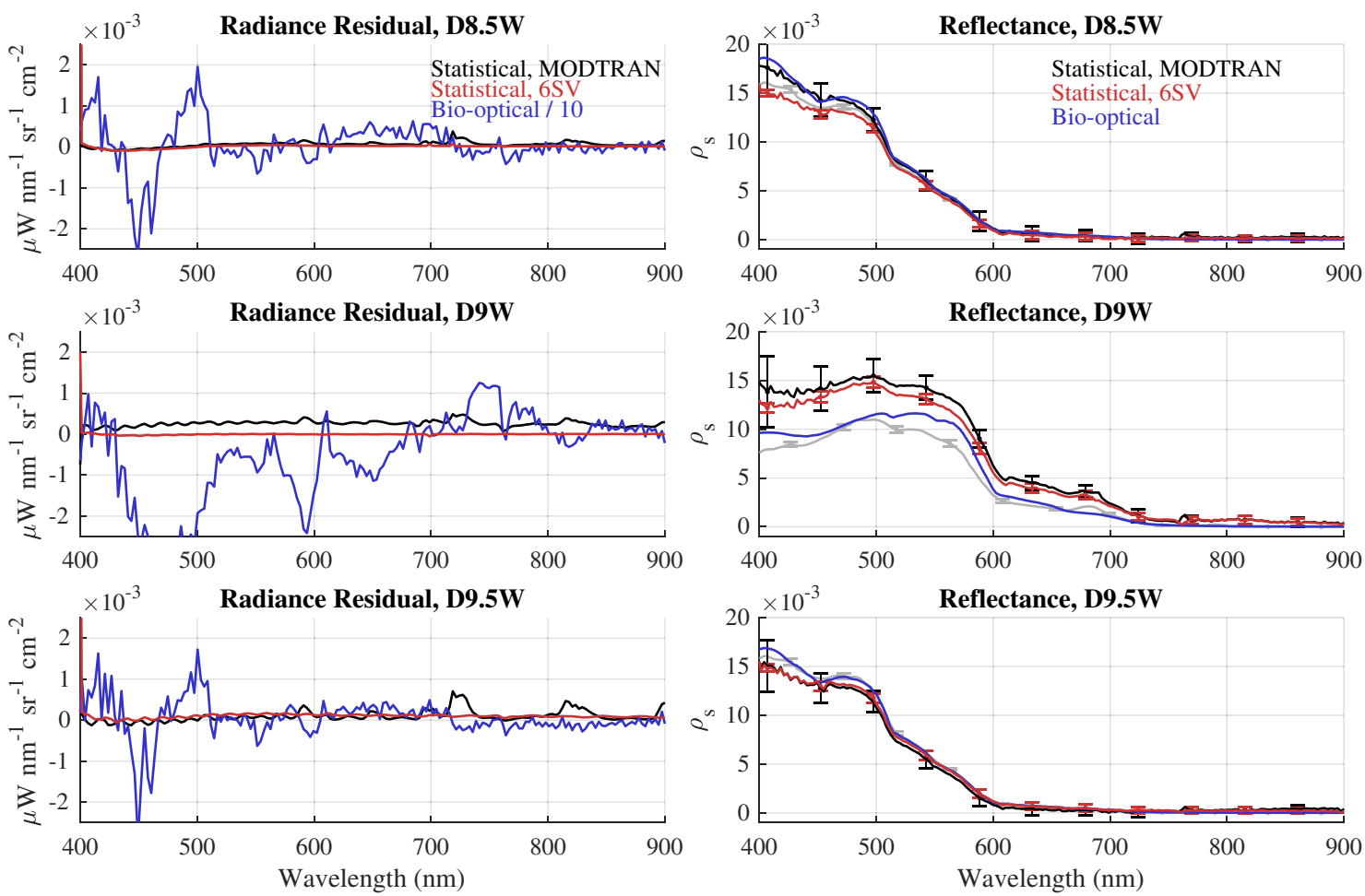

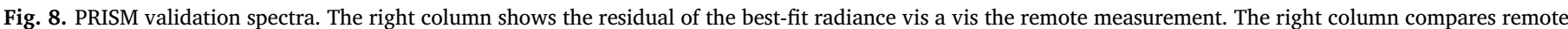

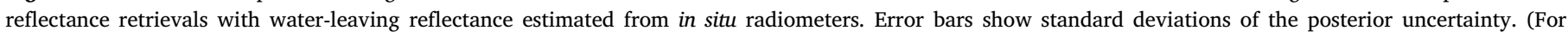
interpretation of the references to color in this figure, the reader is referred to the web version of this article.)
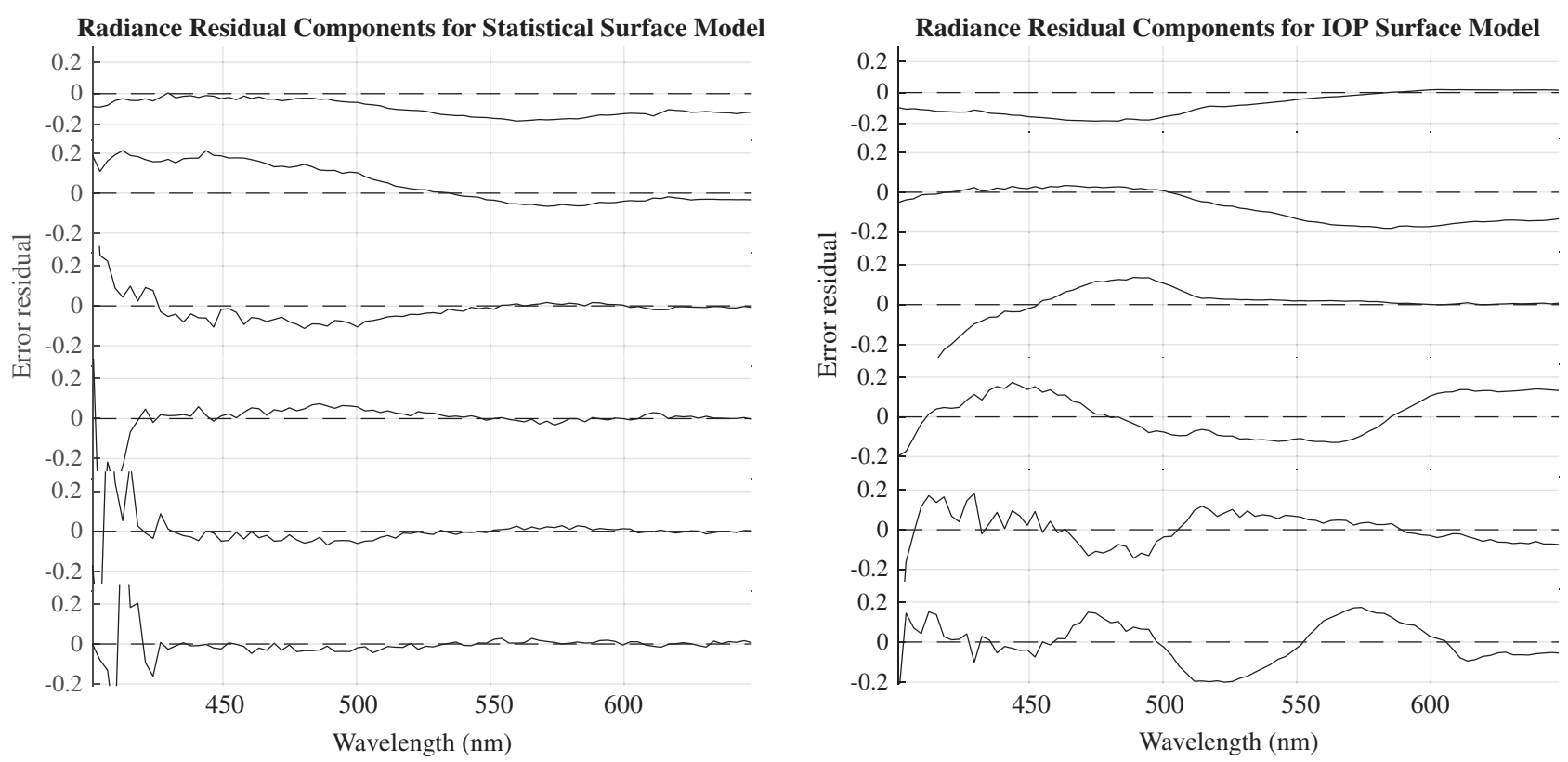

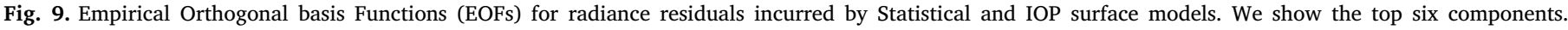
Significance decreases beginning from the top.

and includes both instrument noise and systematic calibration errors per Thompson et al., (2018c). The in situ measurement uncertainty incorporates the temporal variability, again estimated using the empirical time series, as well as a conservative $1 \%$ systematic error term reported by Analytical Spectral Devices, Inc. for the field spectrometers.

\subsection{Experimental results}

Table 5 compares retrievals to field measurements. Spectral angles are similar to the PRISM case, with the OE approach outperforming in $81 \%$ of the cases. Additional columns show the fraction of points exceeding $50 \%$ and $95 \%$ posterior predicted confidence intervals, and the associated $p$ value as before. Extremely high $p$ values indicate the uncertainty budget accounts for observed discrepancies. Fig. 13 shows example spectra from representative field sites. The black, red, blue, and grey lines show the OE solution with the MODTRAN RTM, the OE solution with the 6SV RTM, the standard atmospheric correction, and the in-situ glint corrected surface reflectances respectively. Error bars 

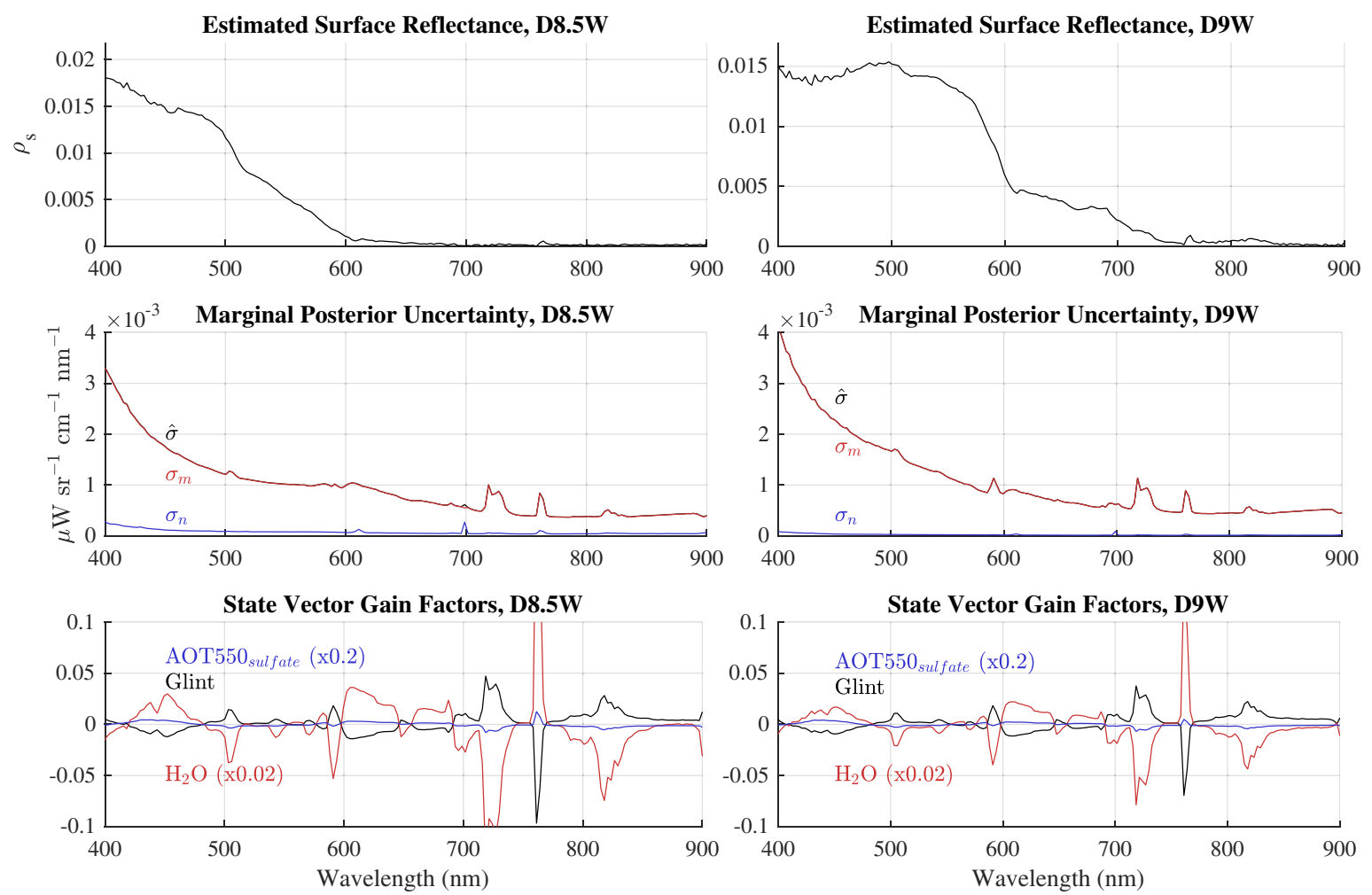

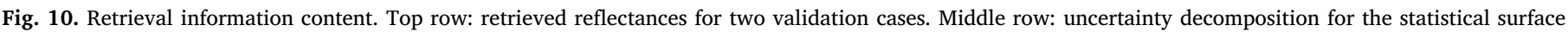

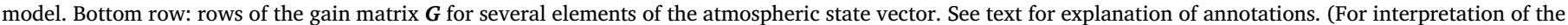
references to color in this figure, the reader is referred to the web version of this article.)

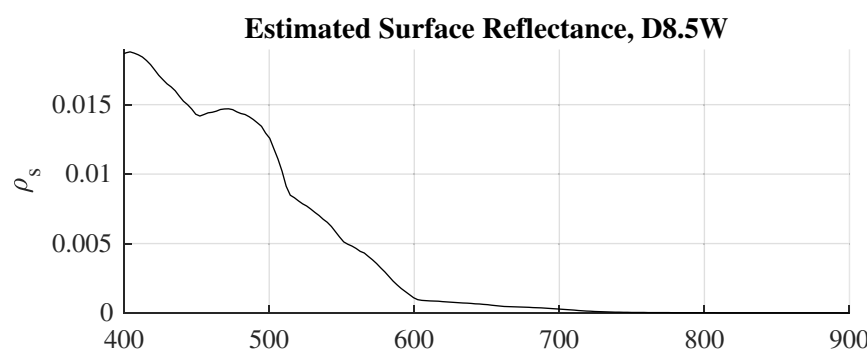

State Vector Gain Factors, D8.5W

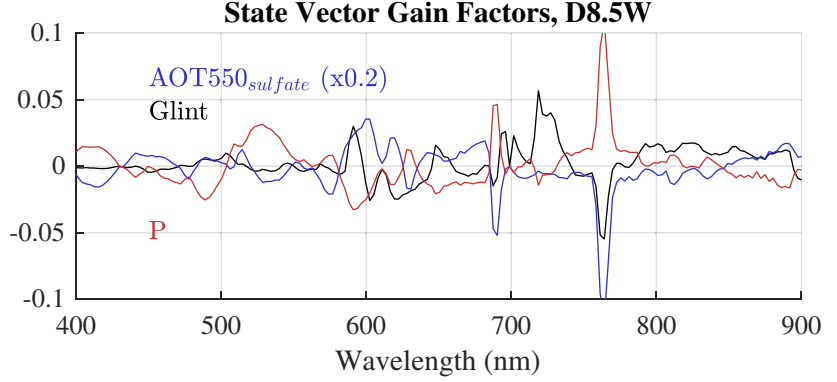

Fig. 11. Gain factors for IOP-based surface parameterization.

indicate the predicted posterior marginal standard deviations. The conventional approach does not provide uncertainty estimates. Qualitatively the results of the different methods are all similar, except in the shortest wavelengths where the OE result better captures the shape though not always the magnitude - of the in situ spectrum. There are also minor differences in the 500 to $700 \mathrm{~nm}$ interval where the $\mathrm{OE}$ approach resolves both 610 and $675 \mathrm{~nm}$ absorptions. The in situ and remote estimates generally align within posterior confidence intervals. Radiance residuals indicate the largest discrepancies in the uncertain blue wavelengths which are most impacted by calibration issues and the sampling uncertainty. The latteris likely responsible for the structured spikes in both RTMs, associated with sharp solar features.

Finally, Fig. 14 shows retrieval error decompositions and gain matrices. The left column shows a turbid open water case. Here, the converged surface reflectance has 196.3 Degrees of Freedom (DOF), slightly lower than the PRISM spectra due to a different balance of Signal to Noise and surface variability. Despite having more channels, AVIRIS-NG has lower signal to noise and the surface reflectance in "extra" channels is highly constrained over water. The right column shows a dark green vegetation spectrum from the scene, which we include as a radically different surface type. The converged reflectance has just 165.8 DOF due to even stronger constraints in vegetation components of the surface model. All atmospheric DOFs exceed 0.99 indicating sensitivity to these parameters. Error decompositions are similar to the PRISM experiment. Water vapor information comes mainly from the atmospheric absorption features, while both visible wavelengths and the modulation of absorption features provide aerosol information. Gain factors grow at long wavelengths due to the lower overall signal. With few exceptions, there is information for all parameters distributed across the entire VSWIR range. The shortwave interval beyond $1500 \mathrm{~nm}$ is particularly discriminative for turbid water; different state vector parameters show very different gain factor profiles in this region. This suggests that slopes and offsets in these spectral intervals can disambiguate the terms.

\section{Discussion and conclusions}

The field experiment reflectance accuracies compare favorably to prior remote spectroscopic study of coastal oceans and inland waters (Jensen et al., 2019; Palacios et al., 2015; Kudela et al., 2015). In 14 validation cases, just one in situ measurement exceeds the posterior predicted error envelope: the PRISM $D 9 W$ site. This was a highly dynamic station close to the effluent outflow, so time variability could contribute to the larger discrepancy. Moreover, there was a time offset 

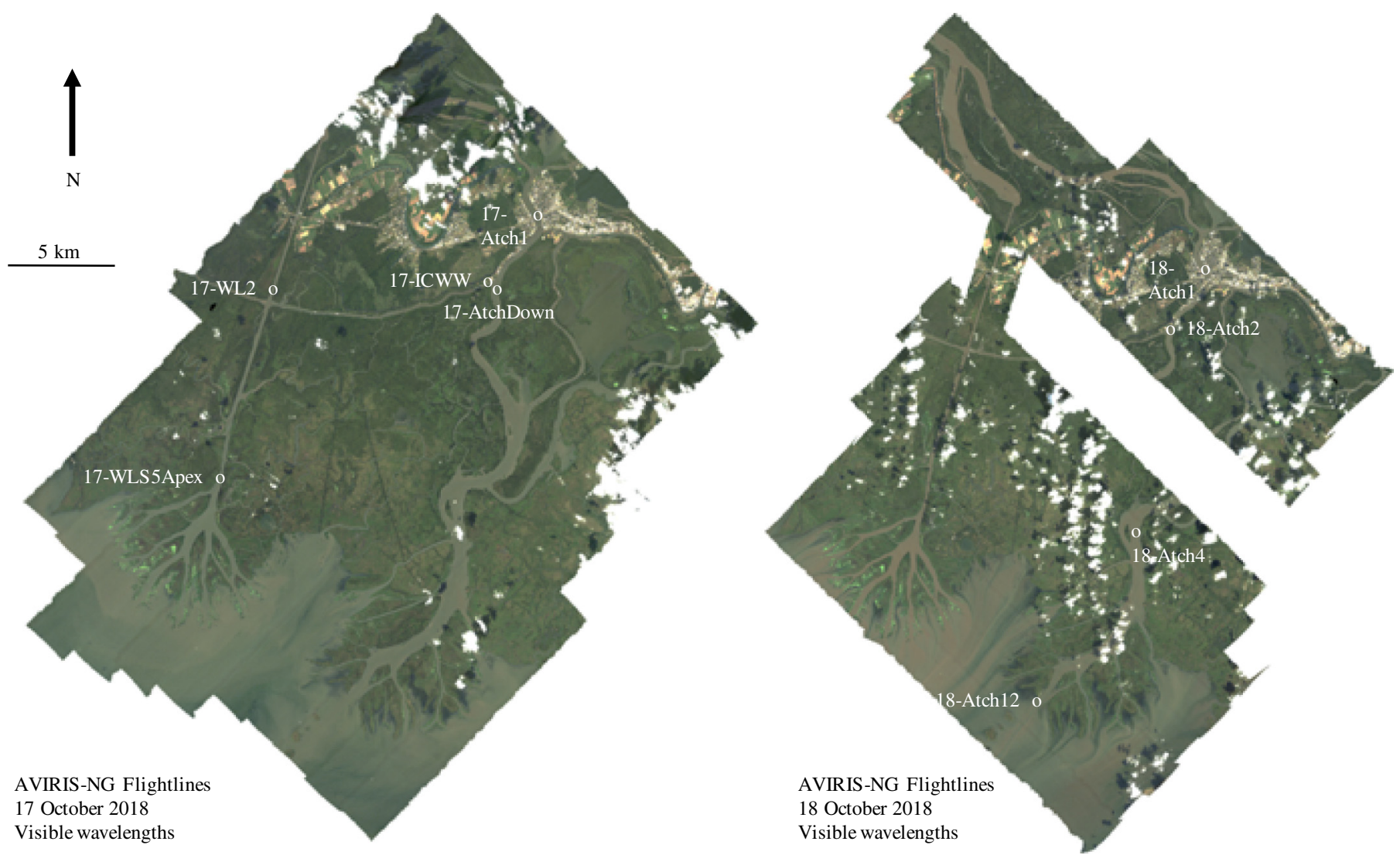

Fig. 12. A mosaic of AVIRIS-NG flightlines covering the Wax Lake Delta and lower Atchafalaya River. The map shows visible wavelength channels and the location of in situ samples on both days. We exclude the vicarious calibration site 17-ICWW from the performance evaluation.

Table 4

Uncertainty account for the AVIRIS-NG campaign. Uncertainty values are standard deviations, except where indicated. We use the channel- and signaldependent AVIRIS-NG measurement uncertainty model from Thompson et al., (2018c). $\mathrm{H}_{2} \mathrm{O}$ uncertainties are consistent with Rothman et al. (2013).

\begin{tabular}{|c|c|c|c|}
\hline Source & Elements & Value & Notes \\
\hline \multicolumn{4}{|l|}{ Remote estimated state $\widehat{\mathbf{x}}$} \\
\hline \multicolumn{4}{|l|}{ Prior uncertainty $\mathbf{S}_{a}$} \\
\hline Surface reflectance & $425 \times 425$ & Empirical & Figs. 2-3 \\
\hline Atmospheric $\mathrm{H}_{2} \mathrm{O}$ & 1 & $>10 \mathrm{~g} \mathrm{~cm}^{-2}$ & \\
\hline Aerosol AOT550 & 3 & 0.05 & \\
\hline \multicolumn{4}{|l|}{ Observation noise $\mathbf{S}_{\mathrm{E}}$} \\
\hline $\begin{array}{l}\text { Measurement noise } \boldsymbol{S}_{\boldsymbol{y}} \\
\text { Unknown variables } \boldsymbol{S}_{b}\end{array}$ & 425 & Varies & See caption \\
\hline $\mathrm{H}_{2} \mathrm{O}$ absorption & 1 & $1 \%$ & See caption \\
\hline \multicolumn{4}{|l|}{ In situ reflectance } \\
\hline Temporal variability & 425 & Varies & Empirical \\
\hline Systematic (calibration) & 1 & $1 \%$ & Manufacturer \\
\hline
\end{tabular}

between the PRISM overpass and the spectrum acquisition at this location, so the uncertainty budget probably underestimates its temporal variability. Unmodeled spatial variability and localization uncertainty may also contribute, since localization error is outside our model. This exception aside, discrepancies are not excessive and most spectrum matches lie well within confidence bounds achieving a consistent closed account of the observation system uncertainty.

Both bio-optical and statistical surface models offer different benefits for coastal aquatic studies. The statistical model consistently achieves the best shape fidelity, with close matches to reference reflectances measured at the surface. It can retrieve features that were not originally incorporated in the source libraries, including subtle modulations of known absorption features by particulate matter and other absorption and packetization that would possibly indicate phytoplankton taxa. It retains the ability to estimate smooth atmospheric parameters using statistical properties of the water-leaving reflectance at long wavelengths. It also permits a unified treatment of terrestrial and aquatic spectra, and - as demonstrated in the AVIRIS-NG flightline - can exploit statistical properties of both to recover spectrally-broad signals. Modelers can apply the degree of regularization which is most appropriate to their application, freely channeling measurement information into atmospheric or surface fidelity. A final benefit is that $\rho_{\text {obs }}$ is nearly linear with respect to the surface state vector, promoting a stable inversion with few local minima. In contrast, the bio-optical model provides direct physical interpretability, automatically interpreting CDOM, chlorophyll-a and fluorescence line height contributions. This may obviate certain downstream analyses and presages more sophisticated parameterizations capable of representing complex water columns. Performing these retrievals in conjunction with atmospheric correction provides a consistent, principled estimate. As an alternative, water leaving reflectance could be propagated to downstream analyses with suitable uncertainty representations (Thompson et al., 2018a, 2018c). These constrained bio-optical surface parameterizations may be more appropriate for low-SNR observations by less capable instruments.

As noted in prior study, different surface types provide varying information with respect to atmospheric phenomena. The entire VSWIR interval proves informative, and AVIRIS-NG exploits shortwave shapes to disambiguate similar sources of interference. Conventional atmospheric correction separates atmosphere and surface heuristically: by bifurcating scenes into land and water areas, further subsetting to dark water and/or dark vegetation, and performing ad hoc tests or optimizations based on slope and magnitude constraints on specific spectral regions. While this can often work well on single flightlines, it requires a large and potentially fragile flowchart of rules that is often tuned 
Table 5

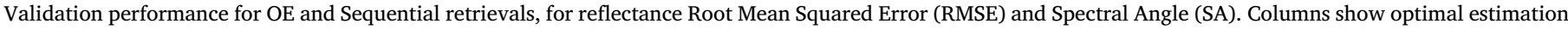

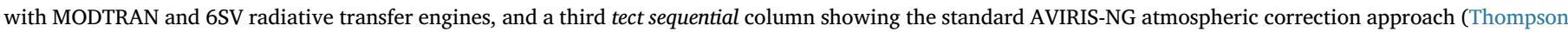

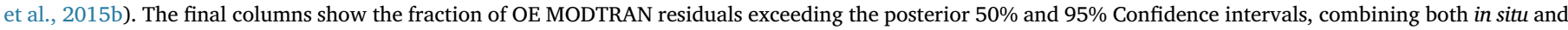
remote posterior uncertainties, and the associated $p$ value.

\begin{tabular}{|c|c|c|c|c|c|c|c|c|c|}
\hline \multirow[t]{2}{*}{ Trial } & \multicolumn{2}{|c|}{ MODTRAN } & \multicolumn{2}{|l|}{$6 S V$} & \multicolumn{2}{|c|}{ Conventional } & \multirow{2}{*}{$\begin{array}{l}>50 \% \\
\text { CI }\end{array}$} & \multirow{2}{*}{$\begin{array}{l}>95 \% \\
\text { CI }\end{array}$} & \multirow[t]{2}{*}{$p$} \\
\hline & RMSE & SA & RMSE & SA & RMSE & SA & & & \\
\hline 17-Atch12 & 0.0042 & 0.088 & 0.0046 & 0.088 & 0.0074 & 0.154 & 38.1 & 0.0 & 1.00 \\
\hline 17-AtchDown & 0.0078 & 0.055 & 0.0124 & 0.076 & 0.0079 & 0.095 & 19.0 & 0.0 & 1.00 \\
\hline 17-Atch1 & 0.0058 & 0.071 & 0.0115 & 0.082 & 0.0092 & 0.164 & 3.8 & 0.0 & 1.00 \\
\hline 17-WLS5Apex & 0.0039 & 0.034 & 0.0123 & 0.151 & 0.0041 & 0.099 & 20.0 & 0.0 & 1.00 \\
\hline 17-WL2 & 0.0034 & 0.026 & 0.0157 & 0.137 & 0.0044 & 0.079 & 20.0 & 0.0 & 1.00 \\
\hline 18-Atch1 & 0.0041 & 0.074 & 0.0105 & 0.103 & 0.0117 & 0.234 & 33.3 & 0.0 & 1.00 \\
\hline 18-Atch2 & 0.0065 & 0.093 & 0.0038 & 0.061 & 0.0069 & 0.130 & 26.7 & 0.0 & 1.00 \\
\hline 18-Atch12 & 0.0068 & 0.134 & 0.0073 & 0.090 & 0.0065 & 0.122 & 27.6 & 0.0 & 1.00 \\
\hline 18-Atch4 & 0.0087 & 0.157 & 0.0104 & 0.121 & 0.0135 & 0.323 & 32.4 & 9.5 & 0.99 \\
\hline 18-WL16 & 0.0076 & 0.247 & 0.0103 & 0.229 & 0.0045 & 0.533 & 21.9 & 2.9 & 1.00 \\
\hline
\end{tabular}
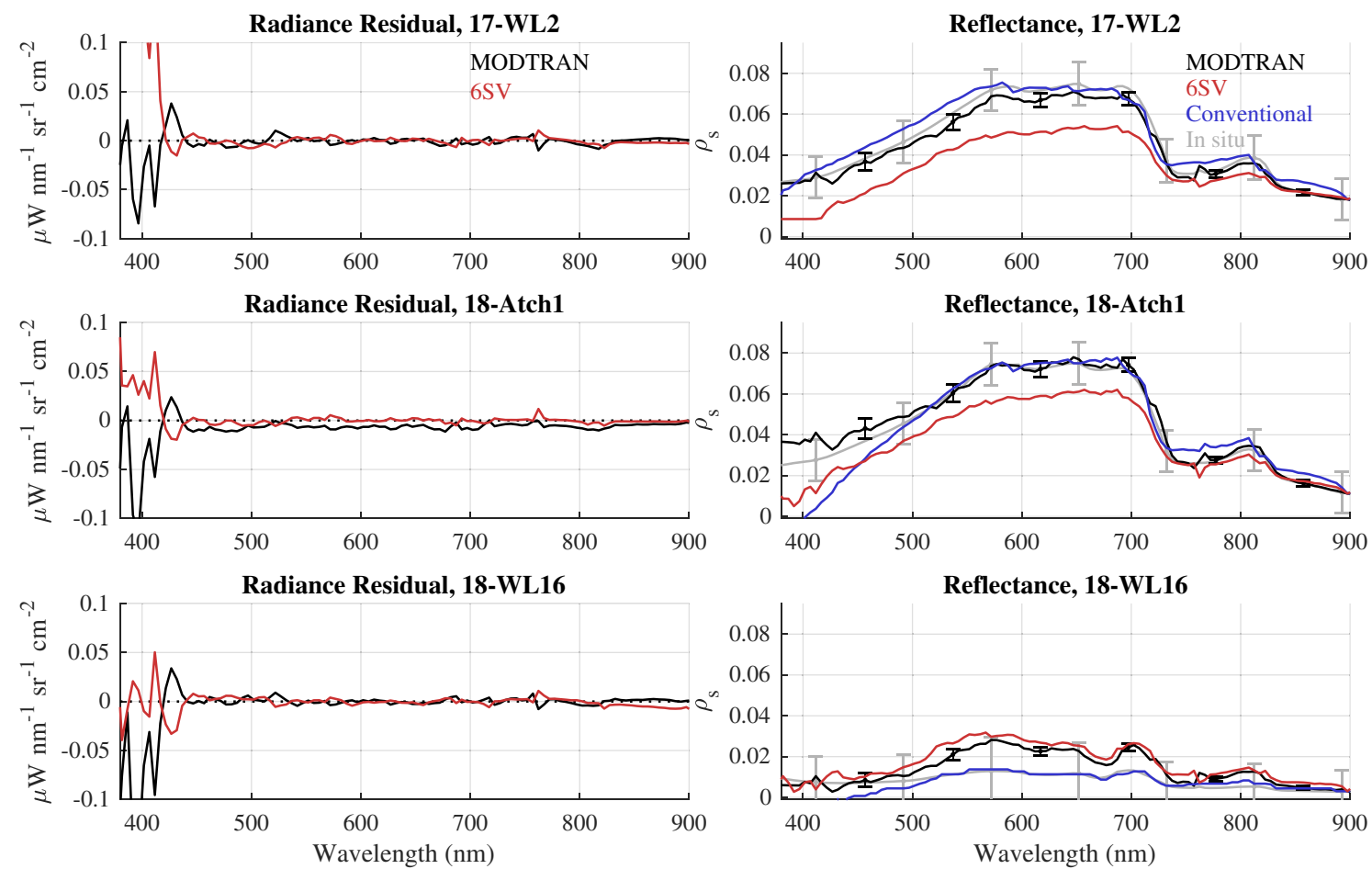

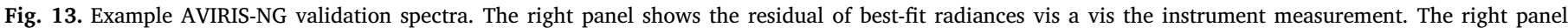

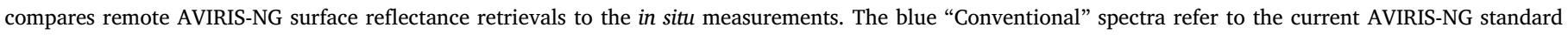

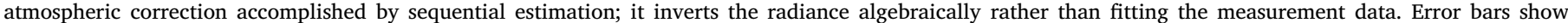

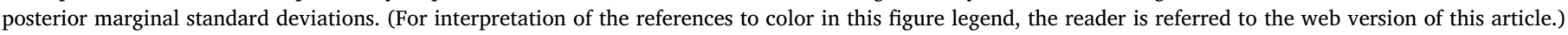

manually to the specific environment . In contrast, $\mathrm{OE}$ automatically captures these constraints through statistics of the surface. It draws appropriate information from all parts of the spectrum without the need for the operators to specify rules in advance. Finally, OE provides a unified framework for land and ocean spectra, enabling complementary information from these different spectra to be combined in a scene-wide atmospheric estimate (Thompson et al., 2018c). Geostatistical techniques, like Gaussian process priors on atmospheric parameters, are a possibility.

There are several other avenues for future study. Neither RTM considered in this experiment is a universal solution for coastal spectroscopy; the 6SV band model carries limited spectral resolution and the MODTRAN code ignores polarization effects. Both decouple the surface and atmosphere estimation, ignoring directional effects of multiple-scattering events from the surface. These simplifications suffice for our experimental demonstration where polarization effects are minimized due to the lack of strong absorption features in the shortest Rayleigh-dominated wavelengths (Natraj et al., 2008), near-nadir observing geometry (Wang, 2006), and instruments designed for polarization insensitivity (Mouroulis et al., 2014). The near-nadir observing angles and low AOD also minimize view-dependent directionality of multiple-scattering from the surface. While these conditions will not always hold, the OE formalism is general enough to accommodate more sophisticated forward models that become available in the future including vector codes with high spectral resolution. One could also couple surface and atmosphere with an RTM that includes both water column and atmosphere simultaneously, resulting in a combined state vector that feeds a new forward model run for each MAP iteration (Fan et al., 2017). Emulation methods (Verrelst et al., 2017; Verrelst et al., 2016) can make this computationally tractable, and it is an area of active ongoing research (Bue et al., 2019).

Future work could also apply the same model to ocean observations. 

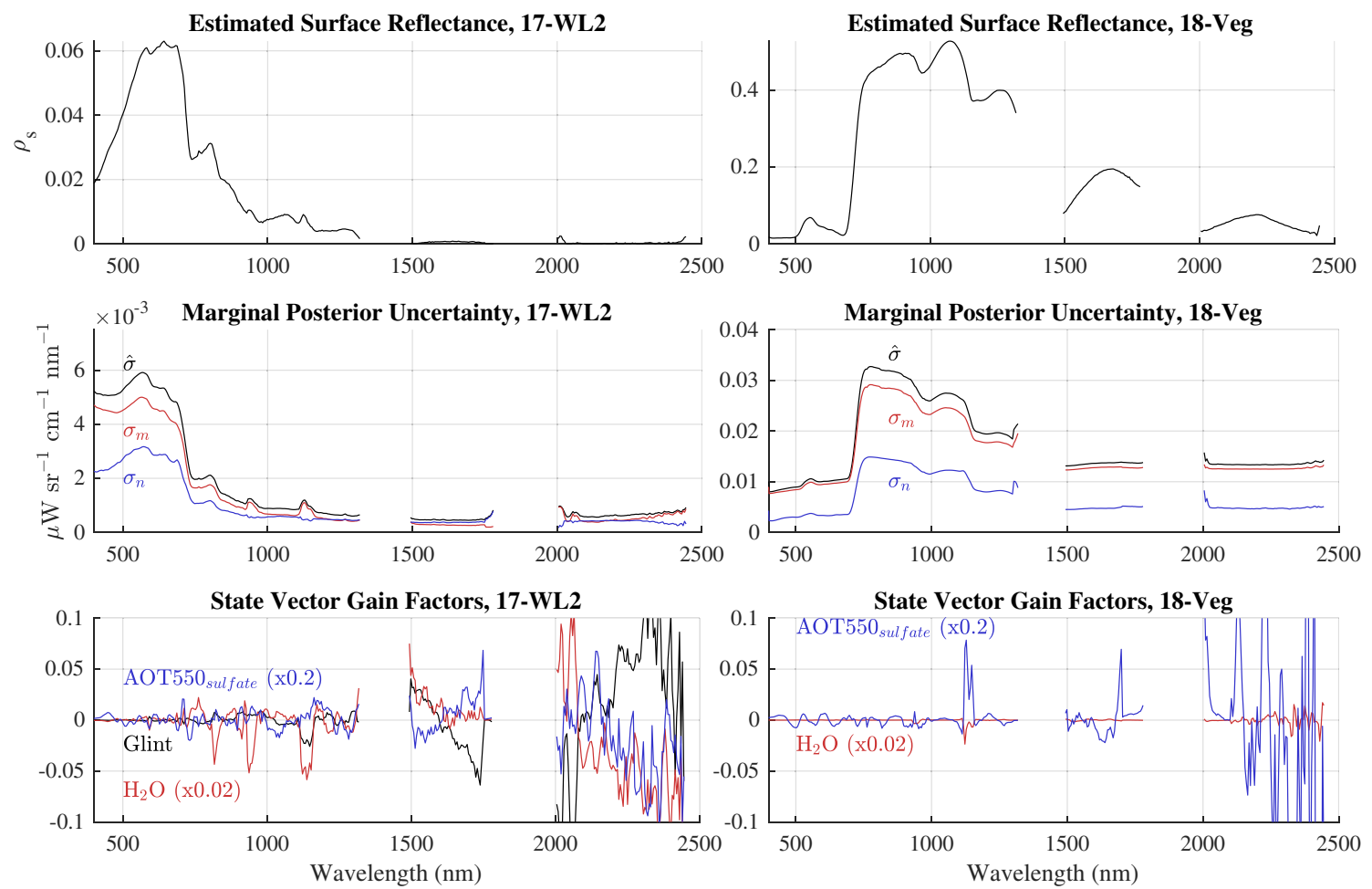

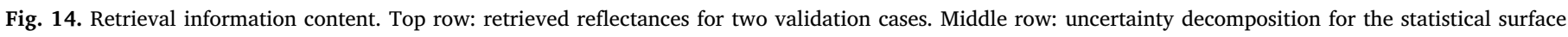

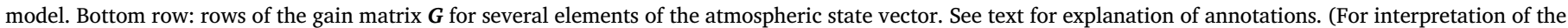
references to color in this figure, the reader is referred to the web version of this article.)

NASA missions like PACE will provide visible/near infrared spectra at coarser spatial resolution over large areas of the open ocean. The Surface Biology and Geology investigation (ESAS, 2018) could cover the entire visible to shortwave infrared interval, through it is likely to focus on terrestrial and coastal areas. In both cases, full-spectrum atmospheric correction algorithms can fully utilize the information present. Global datasets present opportunities for validating $\mathrm{OE}$ approaches using large validation networks imaged multiple times under different atmospheric conditions. New surface models may enable more advanced applications like estimating phytoplankton functional types or discriminating subpixel foam, sea ice, and sea vegetation fractions.

In summary, this study demonstrates that Optimal Estimation methods are applicable to coastal imaging spectroscopy, and hold numerous potential advantages for surface reflectance and atmospheric estimation. We present surface models for clear and turbid water, comparing multiple parameterizations and characterizing the balance in fidelity between surface and atmosphere. An experiment evaluates the methods using NASA's Portable Remote Imaging SpectroMeter (PRISM) over a well-studied wastewater diversion event in Santa Monica, and the Next Generation Airborne Visible Infrared Imaging Spectrometer (AVIRIS-NG) over the Wax Lake Delta and lower Atchafalaya River. The experiments demonstrate the potential of $\mathrm{OE}$ approaches in future coastal spectroscopy studies.

\section{Acknowledgements}

Code used in this study is available through the open source ISOFIT project (Thompson and Olson-Duvall, 2018).

We thank the PRISM team including: Principal Investigator Pantazis Mouroulis; Mark Helmlinger for calibration; Justin Haag for calibration, instrumentation and deployment; Byron Van Gorp for design and development; Frank Loya; and Scott Nolte for operations. The PRISM flight was enabled by a PRISM AITT grant from NASA ESTO. This research was carried out at the Jet Propulsion Laboratory, California Institute of Technology, under a contract with the National Aeronautics and Space Administration and funded under the Presidents' and Directors' Fund Program. We thank other supporting sponsors including the NASA Earth Science Division for the AVIRIS-NG instrument and the data analysis program "Utilization of Airborne Visible/Infrared Imaging Spectrometer Next Generation Data from an Airborne Campaign in India" NNH16ZDA001N-AVRSNG, managed by Woody Turner, for its support of the algorithm development; the Jet Propulsion Laboratory Research and Technology Development Program; and the NASA Center Innovation Fund managed in conjunction with the Jet Propulsion Laboratory Office of the Chief Scientist and Technologist. Copyright 2018 California Institute of Technology. All Rights Reserved. US Government Support Acknowledged.

\section{Appendix A. PRISM instrument noise model}

PRISM is a pushbroom design with a two-dimensional Focal Plane Array (FPA) observing linear segment of the surface. Each cross-track location is essentially a separate spectrometer, and can have slightly different response characteristics. Minor changes in elements' radiometry and spectral responses over the course of a campaign can induce spatially-dependent calibration error manifesting as "striping" in the downtrack spatial dimension. The PRISM optics are designed for high spectral uniformity (Mouroulis et al., 2014) and we assume that wavelength calibration is effectively stable. However, our instrument model does account for variable radiometric response by both a change in the multiplicative correction $\mathbf{c}_{i}$ for each column extiti and noise properties expressed as a column-specific covariance matrix $\mathbf{S}_{y i}$. The radiance $\ell_{o b s}$ relates to the true radiance $\ell_{o b s}{ }^{*}$ by:

$\ell_{o b s}^{*}=\left(\mathbf{c}_{i}^{-1} \circ \ell_{o b s}\right)+\mathbf{S}_{y i}$ 

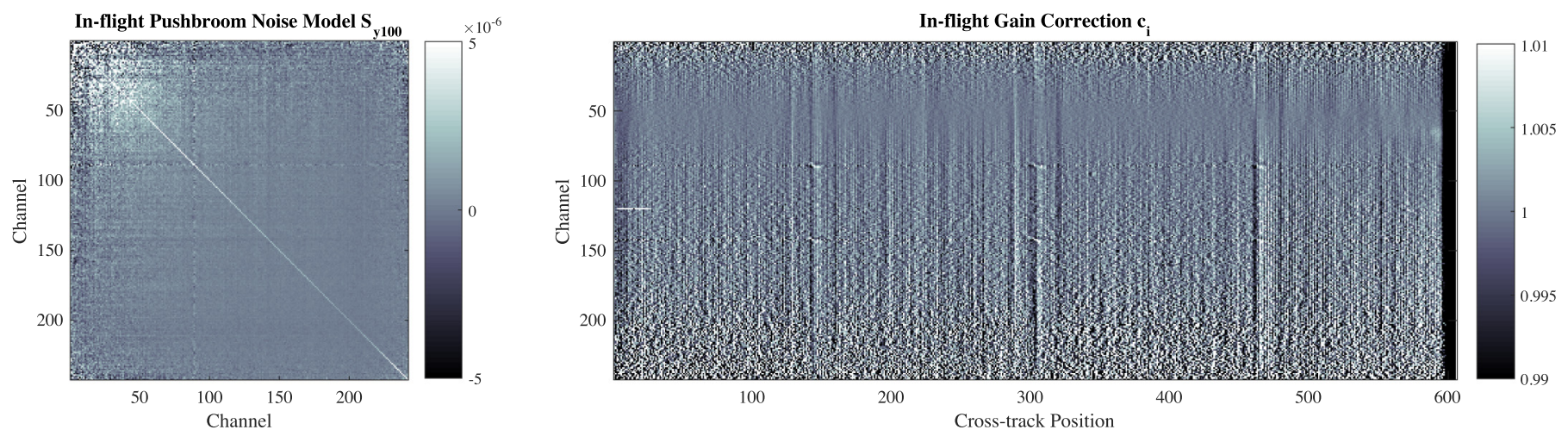

Fig. A.15. Left: Actual estimate for $S_{y i}$ at position 100. Right: matrix comprised of all flat field correction estimates $c_{i}$.

Table A.6

Pushbroom uniformity. $q$ are the quality scores for different retrieval methods. Smaller values are better.

\begin{tabular}{ll}
\hline Method & $q$ \\
\hline Original retrieval with nadir view assumption & 0.003458 \\
Columnwise instrument noise model & 0.002499 \\
Columnwise radiometry correction & 0.001617 \\
Columnwise radiometry and noise & $\mathbf{0 . 0 0 1 5 8 3}$ \\
\hline
\end{tabular}
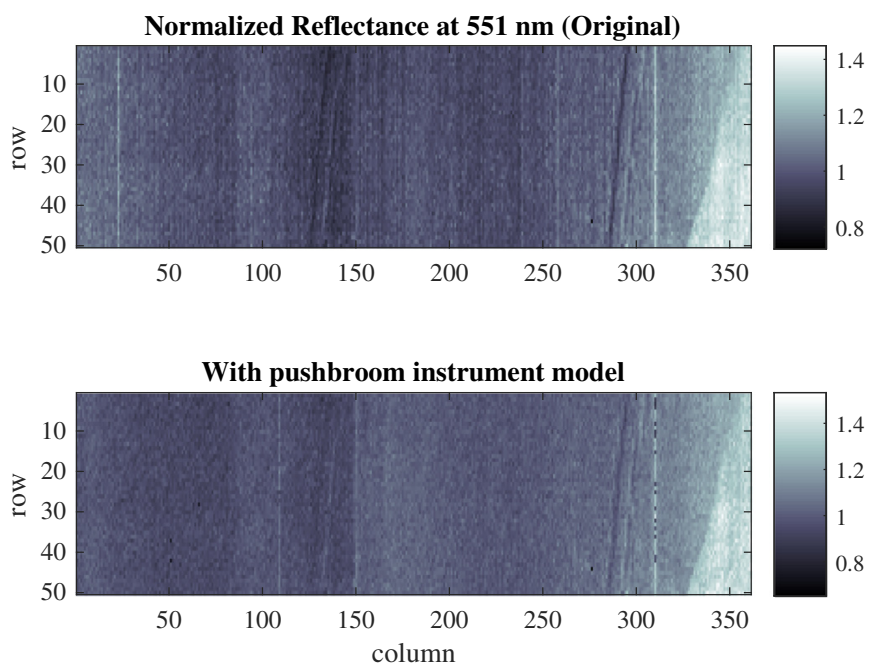

Fig. A.16. The pushbroom instrument model significantly reduces striping effects.

The gain term $c_{i}$ is a vector describing the radiometric response for every channel relative to the other elements; it is a "flat field" value very close to unity. While we always derive initial values for $\mathbf{c}_{i}$ and $\mathbf{S}_{y i}$ using pre-campaign laboratory calibration measurements, there are inevitably tiny changes in each element based on the current thermal and electronic state of the instrument. We exploit the structure of a uniform scene to estimate the values observed in flight. There the instrument flat field contribution is constant within a single column but different across neighboring columns, while the noise contribution is realized independently in each cross-track location and timestep of the acquisition. Any correlated signal likely arises from scene structure rather than the instrument.

These principles allow us to fully decompose the observation variance into the three components of Abbott and Letelier (1999) scene, AdlerGolden et al. (1999) flat field variability, and Amin et al. (2014) instrument noise. Following Thompson et al. (2017), we extract a rectangular region consisting of 100 downtrack pixels and the entire width of the focal plane array. We then apply a simple Gaussian spatial smoothing in the downtrack dimension with a standard deviation of 4 pixels, an operation equivalent to a spatial low pass filter. Subtracting this scene signal from the original acts as a high pass filter leaving the uncorrelated instrument noise. A similar high pass operation in the cross-track dimension reveals the difference in the nominal spectral response of each detector element relative to its neighbors. We express any discrepancy between observed and nominal radiance as a multiplicative gain, and average them downtrack to improve counting statistics. This estimates the gain spectrum $\mathbf{c}_{i}$ for each pushbroom column. Fig. A.15 shows the result: a flat field measurement indicating $\mathbf{c}_{i}$ at all pixels, and a typical covariance matrix $\mathbf{S}_{y i}$. We perform this operation once, before the retrieval. The rate at which updates are required - whether per-flightline, per-day, or per-season - depends on the specifics of the instrument, campaign, and the accuracy needs of the investigation.

We evaluate cross-track uniformity using a performance score $q$ : 
$q=\sum_{\text {bands }} \frac{1}{n_{\text {rows }}} \sum_{\text {rows }} \frac{1}{n_{\text {columns }}} \sum_{\text {columns }}\left(\rho_{\text {si }}-\rho_{\text {sit } 1}\right)^{2}$

where $\rho_{s i}$ and $\rho_{s i+1}$ refer to the surface reflectance of column $i$ and its immediate neighboring column, respectively. The score $q$ estimates the distance between neighboring cross-track locations. This value should be small and average out over long downtrack intervals. Consequently, averaging differences in the downtrack dimension estimates the systematic differences FPA radiometric response. Over large areas, the quality score indicates striping performance. We compare four different accounts of spatial variability: a simple nadir viewing perspective assumption; a model with the viewing geometry of each element; a model with viewing geometry and scene-based instrument noise estimates $\mathbf{S}_{y i}$; and the most sophisticated model with geometry, element-wise noise estimates $\boldsymbol{S}_{y i}$, and element-wise gain adjustments $\boldsymbol{c}_{i}$.

Results indicate that more sophisticated instrument models improve cross track uniformity. Table A.6 reports striping quality scores $q$. Tracking geometry, radiometry and noise independently for each column improves neighbor discrepancies by a factor two relative to the conventional result. Intermediate instrument models incorporating radiometry and noise effects alone achieve partial benefits. Fig. A.16 portrays this improvement, showing the two extreme cases at a representative $550 \mathrm{~nm}$ reflectance channel. The image intentionally stretches visual contrast to emphasize striping effects. Some pathological electronic effects remain in specific columns, but there is a visible reduction in striping.

\section{References}

Abbott, M. R., \& Letelier, R. M. (1999). Algorithm theoretical basis document: chlorophyll fluorescence, MODIS product number 20. NASA Technical Report. Last access 18 June 2018.

Adler-Golden, S.M., Matthew, M.W., Bernstein, L.S., Levine, R.Y., Berk, A., Richtsmeier, S.C., Acharya, P.K., Anderson, G.P., Felde, J.W., Gardner, J., Hokeb, M., Jeongb, L.S., Pukallb, B., Mellob, J., Ratkowskib, A., Burke, H.-H., 1999. Atmospheric correction for shortwave spectral imagery based on modtran4. Imaging Spectrometry V 3753, 61-70.

Amin, R., Lewis, D., Gould, R.W., Hou, W., Lawson, A., Ondrusek, M., Arnone, R., 2014. Assessing the application of cloud-shadow atmospheric correction algorithm on HICO. IEEE Trans. Geosci. Remote Sens. 52, 2646-2653.

Berk, A., Conforti, P., Kennett, R., Perkins, T., Hawes, F., \& van den Bosch, J. (2014). Modtran $^{\circledast}$ 6: a major upgrade of the Modtran ${ }^{\circledast}$ radiative transfer code. (pp. 1-4).

Brajard, J., Moulin, C., Thiria, S., 2008. Atmospheric correction of SeaWifs ocean color imagery in the presence of absorbing aerosols off the Indian coast using a neurovariational method. Geophys. Res. Lett. 35.

Brajard, J., Santer, R., Crépon, M., Thiria, S., 2012. Atmospheric correction of Meris data for case-2 waters using a neuro-variational inversion. Remote Sens. Environ. 126, 51-61.

Bue, B.D., Thompson, D.R., Deshpande, S., Eastwood, M., Fichot, C., Green, R.O., Mullen, T., Natraj, V., Parente, M., 2019. Neural Network Radiative Transfer for Imaging Spectroscopy. Atmos. Meas. Tech. 12, 2567-2578. https://doi.org/10.5194/amt-122567-2019.

Chen, S., Han, L., Chen, X., Li, D., Sun, L., Li, Y., 2015. Estimating wide range total suspended solids concentrations from Modis 250-m imageries: an improved method. ISPRS J. Photogramm. Remote Sens. 99, 58-69.

Corcoran, A.A., Shipe, R.F., 2011. Inshore-offshore and vertical patterns of phytoplankton biomass and community composition in Santa Monica Bay, CA (USA). Estuar. Coast. Shelf Sci. 94, 24-35.

Corson, M.R., Korwan, D.R., Lucke, R.L., Snyder, W.A., Davis, C.O., 2008. The Hyperspectral Imager for the Coastal Ocean (Hico) on the International Space Station. vol. 4 (IV-101).

Cressie, N., 2018. Mission control: a statistical scientist's role in remote sensing of at mospheric carbon dioxide. J. Am. Stat. Assoc. 113, 152-168.

Dubovik, O., Herman, M., Holdak, A., Lapyonok, T., Tanré, D., Deuzé, J., Ducos, F., Sinyuk, A., Lopatin, A., 2011. Statistically optimized inversion algorithm for enhanced retrieval of aerosol properties from spectral multi-angle polarimetric satellite observations. Atmos. Meas. Tech. 4, 975-1018.

ESAS, 2018. Thriving on our Changing Planet: A Decadal Strategy for Earth Observation from Space. A Report by the Decadal Survey on Earth Science and Applications from Space. The National Academies Press.

Fan, Y., Li, W., Gatebe, C.K., Jamet, C., Zibordi, G., Schroeder, T., Stamnes, K., 2017. Atmospheric correction over coastal waters using multilayer neural networks. Remote Sens. Environ. 199, 218-240.

Fichot, C.G., Downing, B.D., Bergamaschi, B.A., Windham-Myers, L., Marvin-DiPasquale, M., Thompson, D.R., Gierach, M.M., 2016. High-resolution remote sensing of water quality in the San Francisco Bay-Delta Estuary. Environ. Sci. Technol. 50, 573-583.

Frouin, R., Pelletier, B., 2015. Bayesian methodology for inverting satellite ocean-color data. Remote Sens. Environ. 159, 332-360.

Frouin, R., et al., 2019. Atmospheric correction of satellite ocean-color imagery during the PACE era. Front. Mar. Sci special issue on the PACE mission (in press).

Gao, B.C., Heidebrecht, K.B., Goetz, A.F., 1993. Derivation of scaled surface reflectances from AVIRIS data. Remote Sens. Environ. 44, 165-178.

Gao, B.C., Montes, M.J., Li, R.R., Dierssen, H.M., Davis, C.O., 2007. An atmospheric correction algorithm for remote sensing of bright coastal waters using Modis land and ocean channels in the solar spectral region. IEEE Trans. Geosci. Remote Sens. 45, 1835-1843.

Gao, B.-C., Montes, M.J., Ahmad, Z., Davis, C.O., 2000. Atmospheric correction algorithm for hyperspectral remote sensing of ocean color from space. Appl. Opt. 39, 887-896.

Giardino, C., Brando, V., Gege, P., Pinnel, N., Hochberg, E., Knaeps, E., Reusen, I., Doerffer, R., Bresciani, M., Braga, F., et al., 2018. Imaging spectrometry of inland and coastal waters: state of the art, achievements and perspectives. Surv. Geophys. 1-29.
Gitelson, A.A., Gao, B.-C., Li, R.-R., Berdnikov, S., Saprygin, V., 2011. Estimation of chlorophyll-a concentration in productive turbid waters using a hyperspectral imager for the coastal ocean - the Azov Sea case study. Environ. Res. Lett. 6, 024023.

Gordon, H.R., 2005. Normalized water-leaving radiance: revisiting the influence of surface roughness. Appl. Opt. 44, 241-248.

Gordon, H.R., Clark, D.K., 1981. Clear water radiances for atmospheric correction of coastal zone color scanner imagery. Appl. Opt. 20, 4175-4180.

Gordon, H.R., Wang, M., 1994. Retrieval of water-leaving radiance and aerosol optical thickness over the oceans with SeaWifs: a preliminary algorithm. Appl. Opt. 33, 443-452.

Gordon, H.R., Brown, O.B., Evans, R.H., Brown, J.W., Smith, R.C., Baker, K.S., Clark, D.K., 1988. A semianalytic radiance model of ocean color. J. Geophys. Res. Atmos. 93, 10909-10924.

Goyens, C., Jamet, C., Schroeder, T., 2013. Evaluation of four atmospheric correction algorithms for MODIS-Aqua images over contrasted coastal waters. Remote Sens. Environ. 131, 63-75.

Green, R.O., Eastwood, M.L., Sarture, C.M., Chrien, T.G., Aronsson, M., Chippendale, B.J., Faust, J.A., Pavri, B.E., Chovit, C.J., Solis, M., et al., 1998. Imaging spectroscopy and the airborne visible/infrared imaging spectrometer (AVIRIS). Remote Sens. Environ. $65,227-248$.

Guanter, L., Segl, K., Sang, B., Alonso, L., Kaufmann, H., Moreno, J., 2009. Scene-based spectral calibration assessment of high spectral resolution imaging spectrometers. Opt. Express 17, 11594-11606.

Hartmann, D.L., Tank, A.M.K., Rusticucci, M., Alexander, L.V., Brönnimann, S., Charabi, Y.A.R., Dentener, F.J., Dlugokencky, E.J., Easterling, D.R., Kaplan, A., et al., 2013. Observations: atmosphere and surface. In: Climate Change 2013 The Physical Science Basis: Working Group I Contribution to the Fifth Assessment Report of the Intergovernmental Panel on Climate Change. Cambridge University Press.

Helmlinger, M., Eastwood, M., Green, R., \& Thompson, D. R. (2016). Solar-similar nearinfra-red suppressed blue calibration source. (pp. 1-11).

Hickey, B.M., 1992. Circulation over the Santa Monica-San Pedro basin and shelf. Prog. Oceanogr. 30, 37-115.

Hochberg, E.J., Atkinson, M.J., Andréfouët, S., 2003. Spectral reflectance of coral reef bottom-types worldwide and implications for coral reef remote sensing. Remote Sens. Environ. 85, 159-173.

Hochberg, E.J., Roberts, D.A., Dennison, P.E., Hulley, G.C., 2015. Special issue on the Hyperspectral InfraRed Imager (HyspIRI): emerging science in terrestrial and aquatic ecology, radiation balance and hazards. Remote Sens. Environ. 167, 1-5.

Hou, W., Wang, J., Xu, X., Reid, J.S., Han, D., 2016. An algorithm for hyperspectral remote sensing of aerosols: 1 . Development of theoretical framework. J. Quant. Spectrosc. Radiat. Transf. 178, 400-415.

Hou, W., Wang, J., Xu, X., Reid, J.S., 2017. An algorithm for hyperspectral remote sensing of aerosols: 2. Information content analysis for aerosol parameters and principal components of surface spectra. J. Quant. Spectrosc. Radiat. Transf. 192, 14-29.

Jensen, D., Simard, M., Cavanaugh, K., Sheng, Y., Fichot, C.G., Pavelsky, T., Twilley, R., Twilley, R., 2019. A comparison of the transferability of empirical multispectral and hyperspectral approaches for estimating suspended solids in wetland and deltaic waters. Remote Sens (in review).

Jensen, D.J., Simard, M., Cavanaugh, K.C., Thompson, D.R., 2018. Imaging spectroscopy BRDF correction for mapping Louisiana's coastal ecosystems. IEEE Trans. Geosci. Remote Sens. 56 (3), 1739-1748.

Kaufman, Y.J., Tanré, D., Remer, L.A., Vermote, E., Chu, A., Holben, B., 1997. Operational remote sensing of tropospheric aerosol over land from EOS moderate resolution imaging spectroradiometer. J. Geophys. Res. Atmos. 102, 17051-17067.

Kneizys, F.X., Shettle, E.P., Abreu, L.W., Chetwynd, J.H., Anderson, G.P., 1988. Users Guide to Lowtran 7. No. AFGL-TR-88-0177, AIR FORCE GEOPHYSICS LAB HANSCOM AFB MA.

Kotchenova, S.Y., Vermote, E.F., 2007. Validation of a vector version of the 6 s radiative transfer code for atmospheric correction of satellite data. Part II. Homogeneous Lambertian and anisotropic surfaces. Appl. Opt. 46, 4455-4464.

Kotchenova, S. Y., Vermote, E. F., Matarrese, R., \& Klemm Jr, F. J. (2006). Validation of a vector version of the $6 \mathrm{~s}$ radiative transfer code for atmospheric correction of satellite data. Part i: path radiance. Appl. Opt., 45, 6762-6774.

Kotchenova, S.Y., Vermote, E.F., Levy, R., Lyapustin, A., 2008. Radiative transfer codes for atmospheric correction and aerosol retrieval: intercomparison study. Appl. Opt. 
47, 2215-2226.

Kudela, R.M., Palacios, S.L., Austerberry, D.C., Accorsi, E.K., Guild, L.S., Torres-Perez, J., 2015. Application of hyperspectral remote sensing to cyanobacterial blooms in inland waters. Remote Sens. Environ. 167, 196-205.

Lee, Z., Carder, K.L., Mobley, C.D., Steward, R.G., Patch, J.S., 1999. Hyperspectral remote sensing for shallow waters: 2 . Deriving bottom depths and water properties by optimization. Appl. Opt. 38, 3831-3843.

Lee, Z., Carder, K.L., Arnone, R.A., 2002. Deriving inherent optical properties from water color: a multiband quasi-analytical algorithm for optically deep waters. Appl. Opt. $41,5755-5772$.

Lee, Z., Carder, K.L., Du, K., 2004. Effects of molecular and particle scatterings on the model parameter for remote-sensing reflectance. Appl. Opt. 43, 4957-4964.

Lewis, M.D., Gould, R., Arnone, R., Lyon, P., Martinolich, P., Vaughan, R., Lawson, A. Scardino, T., Hou, W., Snyder, W., et al., 2009. The Hyperspectral Imager for the Coastal Ocean (Hico): Sensor and Data Processing Overview. pp. 1-9.

Mobley, C. D., Werdell, J., Franz, B., Ahmad, Z., \& Bailey, S. (2016). Atmospheric Correction for Satellite Ocean Color Radiometry. NASA Technical Report, NASA/ TM2016-217551. Available from https://ntrs.nasa.gov/search.jsp?R=20160011399, Last access 26 May 2018.

More, J.J., 1978. The Levenberg-Marquardt algorithm: implementation and theory. Numerical Analysis 105-116.

Morel, A., 1974. Optical properties of pure water and pure sea water. In: Optical Aspects of Oceanography, pp. 1-24.

Morris, J.T., Sundareshwar, P.V., Nietch, C.T., Kjerfve, B., 2002. Responses of coastal wetlands to rising sea level. Ecology 83, 2869-2877.

Mouroulis, P., Van Gorp, B., Green, R.O., Dierssen, H., Wilson, D.W., Eastwood, M., Boardman, J., Gao, B.-C., Cohen, D., Franklin, B., et al., 2014. Portable remote imaging spectrometer coastal ocean sensor: design, characteristics, and first flight results. Appl. Opt. 53, 1363-1380.

Muller-Karger, F.E., Hestir, E., Ade, C., Turpie, K., Roberts, D.A., Siegel, D., Miller, R.J., Humm, D., Izenberg, N., Keller, M., et al., 2018. Satellite sensor requirements for monitoring essential biodiversity variables of coastal ecosystems. Ecol. Appl. 28, 749-760.

Natraj, V., Boesch, H., Spurr, R.J., Yung, Y.L., 2008. Retrieval of from simulated orbiting carbon observatory measurements using the fast linearized $r$-2os radiative transfer model. J. Geophys. Res. Atmos. 113.

Neumann, A., Doerffer, R., Krawczyk, H., Dowell, M.D., Arnone, R., Davis, C.O., Kishino, M., Tanaka, A., Hu, C., Bukata, R.P., Gordon, H.R., Campbell, J., Sathyendranath, S., 2000. Algorithms for case 2 waters. In: Sathyendranath, S. (Ed.), Reports of the International Ocean-Colour Coordinating Group, IOCCG Report 3: Remote Sensing of Ocean Colour in Coastal, and Other Optically-Complex, Waters. MacNab Print, Dartmouth, Canada, pp. 47 chapter 3.

Palacios, S.L., Kudela, R.M., Guild, L.S., Negrey, K.H., Torres-Perez, J., Broughton, J., 2015. Remote sensing of phytoplankton functional types in the coastal ocean from the hyspiri preparatory flight campaign. Remote Sens. Environ. 167, 269-280.

Parslow, J.S., Hoepffner, N., Doerffer, R., Campbell, J.W., Schlittenhardt, P., Sathyendranath, S., 2000. Case 2 ocean-colour applications. In: Sathyendranath, S. (Ed.), Reports of the International Ocean-Colour Coordinating Group, IOCCG Report 3: Remote Sensing of Ocean Colour in Coastal, and Other Optically-Complex, Waters Chapter 5. MacNab Print, Dartmouth, Canada, pp. 93.

Perkins, T., Adler-Golden, S., Matthew, M., Berk, A., Bernstein, L., Lee, J., et al., 2012. Speed and accuracy improvements in FLAASH atmospheric correction of hyperspectral imagery. Opt. Eng. 51, 111707-1-111707-7.

Richter, R., Schläpfer, D., 2002. Geo-Atmospheric Processing of Airborne Imaging Spectrometry Data, Part 2: Atmospheric/Topographic Correction.

Richter, R., Schläpfer, D., 2005. Atmospheric/topographic correction for satellite imagery. In: DLR Report DLR-IB, pp. 565-601.

Rodgers, C.D., 2000. Inverse Methods for Atmospheric Sounding: Theory and Practice Volume 2. World Scientific.

Rothman, L.S., Gordon, I.E., Babikov, Y., Barbe, A., Chris Benner, D., Bernath, P.F., Birk, M., Bizzocchi, L., Boudon, V., Brown, L.R., et al., 2013. The hitran2012 molecular spectroscopic database. J. Quant. Spectrosc. Radiat. Transf. 130, 4-50.

Saulquin, B., Fablet, R., Bourg, L., Mercier, G., d'Andon, O.F., 2016. MEETC2: ocean color atmospheric corrections in coastal complex waters using a bayesian latent class model and potential for the incoming sentinel 3-OLCI mission. Remote Sens. Environ. 172, 39-49.

Schaepman, M.E., Ustin, S.L., Plaza, A.J., Painter, T.H., Verrelst, J., Liang, S., 2009. Earth system science related imaging spectroscopy —an assessment. Remote Sens. Environ. 113, S123-S137.

Schläpfer, D., Borel, C.C., Keller, J., Itten, K.I., 1998. Atmospheric precorrected differential absorption technique to retrieve columnar water vapor. Remote Sens. Environ. 65, 353-366.

Siegel, D.A., Wang, M., Maritorena, S., Robinson, W., 2000. Atmospheric correction of satellite ocean color imagery: the black pixel assumption. Appl. Opt. 39, 3582-3591.

Spurr, R.J., 2006. Vlidort: a linearized pseudo-spherical vector discrete ordinate radiative transfer code for forward model and retrieval studies in multilayer multiple scattering media. J. Quant. Spectrosc. Radiat. Transf. 102, 316-342.

Stamnes, K., Tsay, S.-C., Wiscombe, W., Jayaweera, K., 1988. Numerically stable algorithm for discrete-ordinate-method radiative transfer in multiple scattering and emitting layered media. Appl. Opt. 27, 2502-2509.

Steinmetz, F., Deschamps, P.-Y., Ramon, D., 2011. Atmospheric correction in presence of sun glint: application to Meris. Opt. Express 19, 9783-9800.

Tan, J., Cherkauer, K.A., Chaubey, I., 2016. Developing a comprehensive spectral-biogeochemical database of Midwestern rivers for water quality retrieval using remote sensing data: a case study of the Wabash River and its tributary, Indiana. Remote Sens. 8.

Theiler, J., 2012. The incredible shrinking covariance estimator. In: SPIE Defense, Security, and Sensing. International Society for Optics and Photonics, pp. 83910P.

Thompson, D., Leifer, I., Bovensmann, H., Eastwood, M., Fladeland, M., Frankenberg, C., Gerilowski, K., Green, R., Kratwurst, S., Krings, T., et al., 2015a. Real-time remote detection and measurement for airborne imaging spectroscopy: a case study with methane. Atmos. Meas. Tech. 8, 4383-4397.

Thompson, D.R., Olson-Duvall, W., 2018. Imaging Spectrometer Optimal FITting (ISOFIT). GitHub repository, released under the Apache 2.0 open source license. https://github.com/davidraythompson/isofit.

Thompson, D.R., Gao, B.-C., Green, R.O., Roberts, D.A., Dennison, P.E., Lundeen, S.R. 2015b. Atmospheric correction for global mapping spectroscopy: ATREM advances for the HyspIRI preparatory campaign. Remote Sens. Environ. 167, 64-77.

Thompson, D.R., Roberts, D.A., Gao, B.C., Green, R.O., Guild, L., Hayashi, K., Kudela, R., Palacios, S., 2016. Atmospheric correction with the Bayesian empirical line. Opt. Express 24, 2134-2144.

Thompson, D.R., Boardman, J.W., Eastwood, M.L., Green, R.O., 2017. A large airborne survey of earth's visible-infrared spectral dimensionality. Opt. Express 24.

Thompson, D.R., Boardman, J.W., Eastwood, M.L., Green, R.O., Haag, J.M., Mouroulis, P., Gorp, B.V., 2018a. Imaging spectrometer stray spectral response: in-flight characterization, correction, and validation. Remote Sens. Environ. 204, 850-860.

Thompson, D.R., Guanter, L., Berk, A., Gao, B.-C., Richter, R., Schläpfer, D., Thome, K.J., 2018b. Retrieval of atmospheric parameters and surface reflectance from imaging spectroscopy data. Surv. Geophys. 40, 333-360 (in press).

Thompson, D.R., Natraj, V., Green, R.O., Helmlinger, M., Gao, B.-C., Eastwood, M., 2018c Optimal estimation for imaging spectrometer atmospheric correction. Remote Sens. Environ. 216, 355-373.

Trinh, R.C., Fichot, C.G., Gierach, M.M., Holt, B., Malakar, N.K., Hulley, G., Smith, J., 2017. Application of Landsat 8 for monitoring impacts of wastewater discharge on coastal water quality. Front. Mar. Sci. 4.

Turpie, K.R., Klemas, V.V., Byrd, K., Kelly, M., Jo, Y.-H., 2015. Prospective HyspIRI global observations of tidal wetlands. Remote Sens. Environ. 167, 206-217.

Twilley, R.R., Bentley, S.J., Chen, Q., Edmonds, D.A., Hagen, S.C., Lam, N.S.-N., Willson, C.S., Xu, K., Braud, D., Peele, R.H., et al., 2016. Co-evolution of wetland landscapes, flooding, and human settlement in the Mississippi River delta plain. Sustain. Sci. 11, 711-731.

Vermote, E.F., Kotchenova, S., 2008. Atmospheric correction for the monitoring of land surfaces. J. Geophys. Res. Atmos. 113.

Vermote, E.F., Tanré, D., Deuze, J.L., Herman, M., Morcette, J.-J., 1997. Second simulation of the satellite signal in the solar spectrum, 6s: an overview. IEEE Trans, Geosci. Remote Sens. 35, 675-686.

Vermote, E.F., El Saleous, N.Z., Justice, C.O., 2002. Atmospheric correction of modis data in the visible to middle infrared: first results. Remote Sens. Environ. 83, 97-111.

Verrelst, J., Sabater, N., Rivera, J.P., Muñoz-Marí, J., Vicent, J., Camps-Valls, G., Moreno, J., 2016. Emulation of leaf, canopy and atmosphere radiative transfer models for fast global sensitivity analysis. Remote Sens. 8, 673.

Verrelst, J., Rivera Caicedo, J., Muñoz-Marí, J., Camps-Valls, G., Moreno, J., 2017. Scopebased emulators for fast generation of synthetic canopy reflectance and sun-induced fluorescence spectra. Remote Sens. 9, 927.

Wang, M., 2006. Aerosol polarization effects on atmospheric correction and aerosol retrievals in ocean color remote sensing. Appl. Opt. 45, 8951-8963.

Wang, M., Shi, W., 2007. The NIR-SWIR combined atmospheric correction approach for modis ocean color data processing. Opt. Express 15, 15722-15733.

Wang, M., Antoine, D., Frouin, R., Gordon, H.R., Fukushima, H., Morel, A., Nicolas, J.-M., Deschamps, P.-Y., 2010. In: Wang, M. (Ed.), Reports of the International OceanColour Coordinating Group, IOCCG Report 10: Atmospheric Correction for RemotelySensed Ocean-Colour Products. MacNab Print, Dartmouth, Canada, pp. 23 chapter 4.

Warrick, J.a., Mertes, L.a.K., Siegel, D.a., Mackenzie, C., 2004. Estimating suspended sediment concentrations in turbid coastal waters of the Santa Barbara channel with seawifs. Int. J. Remote Sens. 25, 1995-2002. 